\title{
Title: Experience-Driven Rate Modulation is Reinstated During Hippocampal Replay
}

\author{
Authors: M. Tirole ${ }^{1 * \dagger}$, M. Huelin Gorriz ${ }^{1 * \dagger}$, M. Takigawa ${ }^{1}$, L. Kukovska $^{1}$, D. Bendor ${ }^{1}$.
}

\author{
Affiliations: \\ ${ }^{1}$ Institute of Behavioural Neuroscience (IBN), University College London (UCL); London, \\ WC1H 0AP, UK \\ *Co-corresponding authors. (margot.tirole.14@ucl.ac.uk, marta.huelin.16@ucl.ac.uk) \\ $\uparrow$ These authors contributed equally
}

\begin{abstract}
Replay, the sequential reactivation of a neuronal ensemble, is thought to play a central role in the hippocampus during the consolidation of a recent experience into a long-term memory. Following a contextual change (e.g. entering a novel environment), hippocampal place cells typically modulate their in-field firing rate and shift the position of their place field, providing a rate and place representation for the behavioral episode, respectively. However, replay has been largely defined by only the latter- based on the fidelity of sequential activity across neighboring place fields. Here we show that dorsal CA1 place cells in rats can modulate their firing rate between the replay of two different contexts, mirroring the same pattern of rate modulation observed during behavior. This context-driven rate modulation within replay events was experience-dependent, observable during both behavioral episodes and throughout the subsequent rest period, but not prior to experience. Furthermore, we demonstrate that both the temporal order and firing rate of place cells can independently be used to decode contextual information within a replay event, revealing the existence of two separable but complementary neural representations available for memory consolidation processes.
\end{abstract}




\section{Main Text:}

A key function of the hippocampus is the initial encoding and subsequent consolidation of episodic memories. Hippocampal place cells are activated in sequential patterns during behavioral episodes (e.g. a rodent running towards a goal), with each neuron's activity modulated by the animal's position within an environment (i.e. a place field) (1). During offline periods such as sleep, place cells spontaneously reactivate, replaying the same sequential pattern previously activated during the behavioral episode $(2,3)$. This phenomenon of neural replay is postulated to drive systems-level memory consolidation (4-6). Hippocampal replay has largely been defined by the sequential reactivation of place cell ensembles $(3,7-9)$, driven by both where and when each place field is activated during a behavioral episode (here referred to as place representation). Yet, place cells also carry additional information in the magnitude of their place field's activity (here referred to as rate representation). The magnitude of a place field's activity (i.e. peak in-field firing rate) is modulated by both local and global contextual cues (1012), as well as behavioral variables (e.g. animal's speed during locomotion) (13), and cognitive events (e.g. animal's attention and perception) $(14,15)$. However, we do not know whether the hippocampus is capable of reinstating this rate modulation in place cells during offline replay.

To address this question, we recorded extracellularly from the dorsal CA1 of rats $(n=3$, male Lister-hooded), trained to run back and forth along two novel linear tracks $(2 \mathrm{~m})$ to collect a liquid reward at each end (Fig. 1A, fig. S1A, table S1, and supplementary methods). A viewobstructing divider was placed between tracks and distinguishing visual cues were present within each environment to facilitate contextual discrimination and, in turn, hippocampal remapping. Additionally, rats rested in a quiet remote location, both before (PRE) and after (POST) the exploration of the two tracks.

We observed global remapping of place cells between the two linear tracks [Pearson correlation coefficient $(r)$ between tracks population vectors, $r=0.04 \pm 0.11]$, such that the majority of place fields shifted their location along the track and/or modulated their firing rate (i.e. changed to higher or lower peak in-field firing rate) (fig. S1B). Both the context (which linear track) and current position (location of the animal on the linear track) could be inferred from place cell activity with high accuracy using a naïve Bayes decoder (Fig. 1B and fig.S1C). Track detection accuracy exceeded $89 \%$ during RUN periods on both tracks [all sessions: $89 \pm 5 \%$ ] with an average median error of $5 \mathrm{~cm}$ [all sessions: $5.17 \pm 0.69 \mathrm{~cm}$ ]. A population vector analysis showed high correlation between the ratemaps of the first and second half of running within each track [Pearson correlation coefficient $(r)$ within track population vectors, $r=0.83 \pm 0.04$ ], suggesting high place field stability over run. We also used Bayesian decoding to detect replay sequences of each linear track (Fig. 1B), with statistically significant replay trajectories identified by comparing the weighted correlation score obtained from the posterior probabilities of each decoded event to three types of event shuffles [Total replay events $=2805$, PRE: Track $1=35 \pm 20$, Track2 $=37 \pm 15$, POST: Track $1=138 \pm 61$, Track2 $=156 \pm 34$, RUN: Track $1=98 \pm 20$, Track2= $95 \pm 31$; fig. S2 and supplementary methods]. 


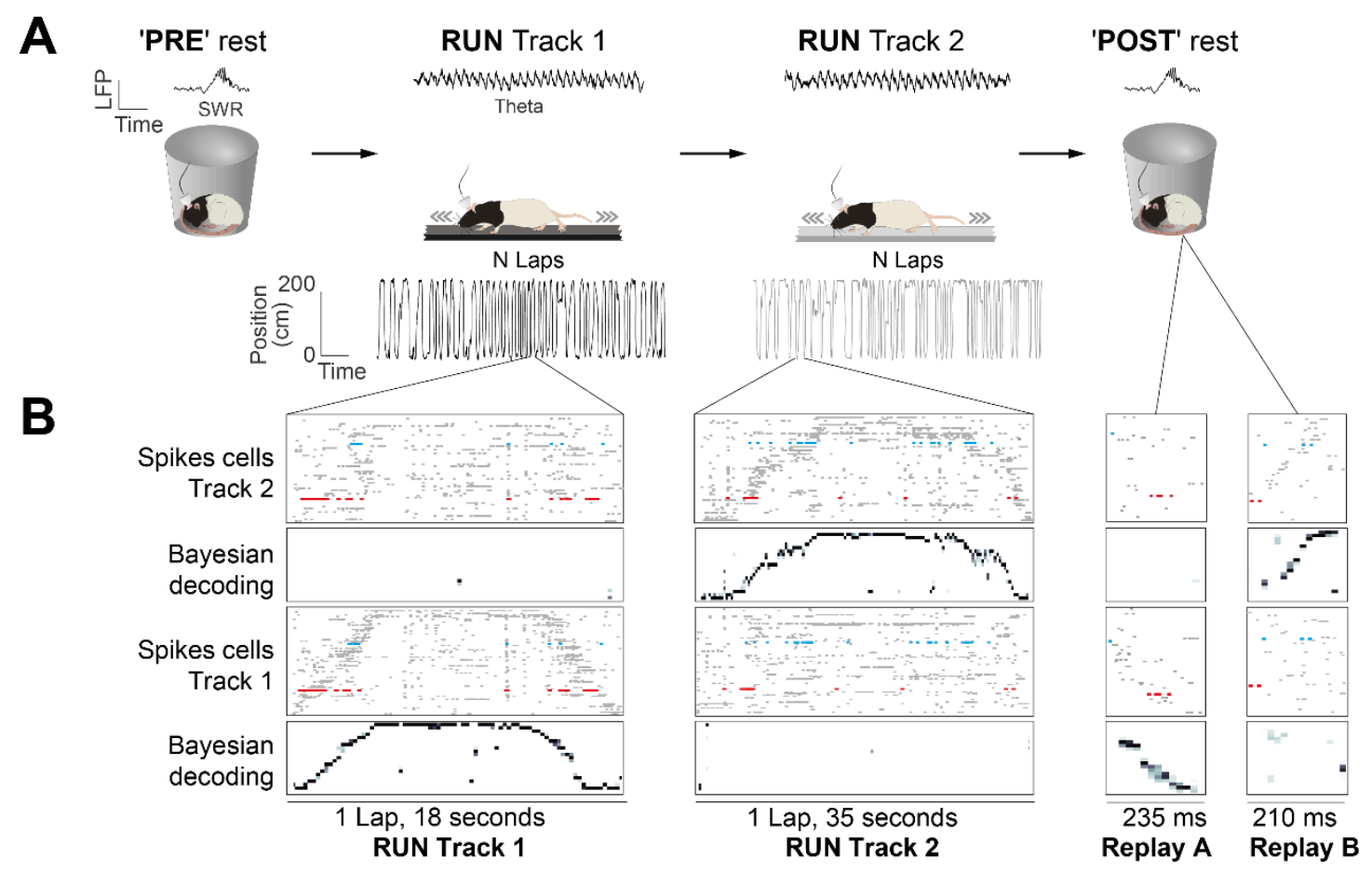

C

Cell ID: 5097

Cell ID: 5029
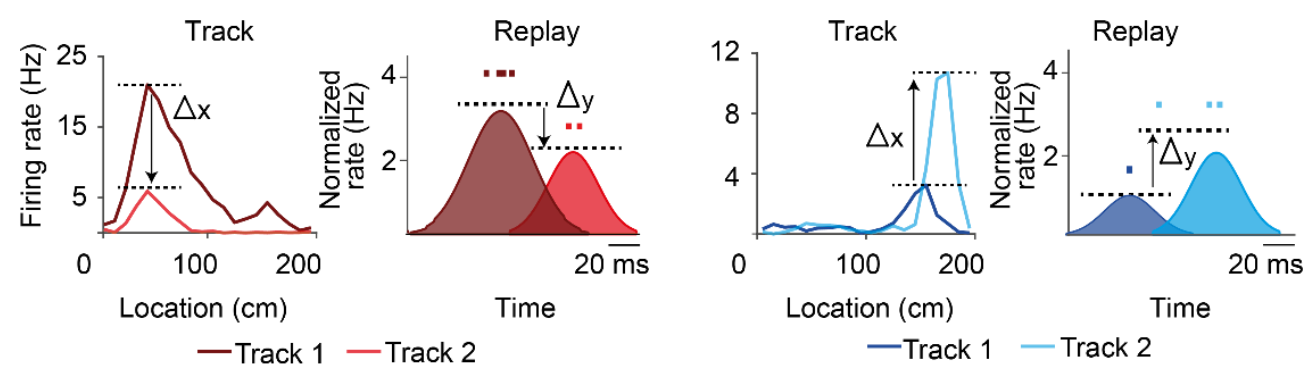

D
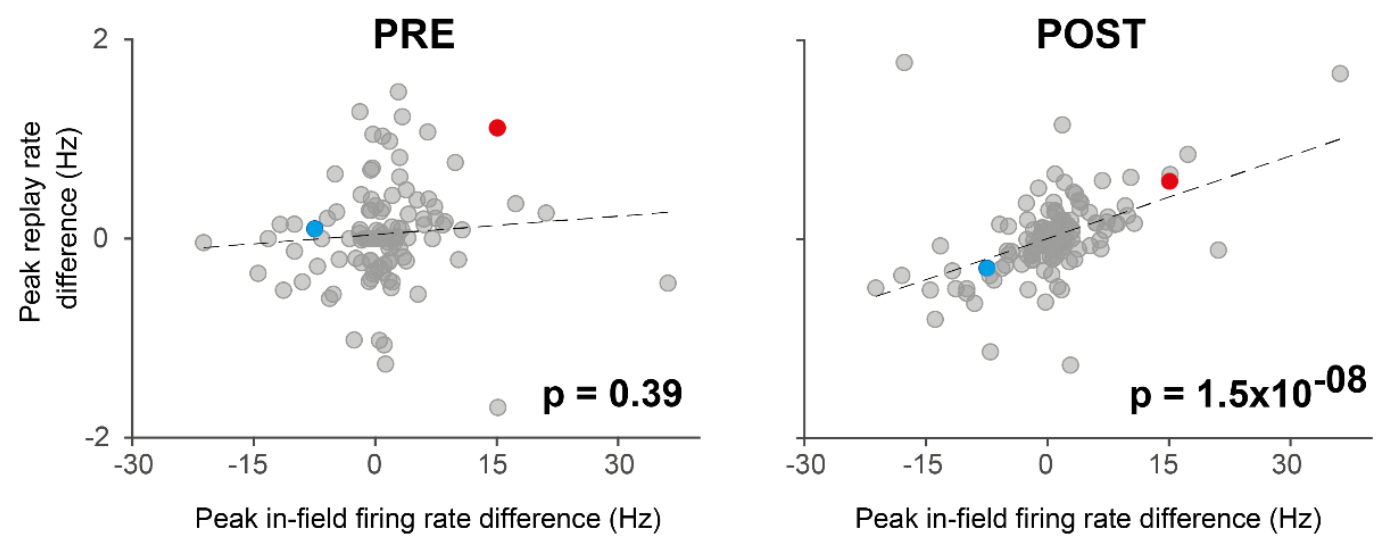

Fig. 1. Rate coding in wake and rest. (A) Experimental design. Each recording session, the rats rested (PRE), ran back and forth on two different linear tracks (RUN), and then rested again (POST) (left to right). An example of LFP activity in each behavioral state is displayed [RUNtheta, PRE/POST-sharp wave ripples (SWR)]. (B) Raster plots of spiking activity of cells sorted by their peak firing rate on the track (top: Track $2, \mathrm{n}=57$ cells; bottom: Track $1, \mathrm{n}=72$ cells), and the associated decoded positions for a single exemplar lap (first two columns) and replay 
event (last two columns), for each track (top: Track 2; bottom: Track 1). Example from Rat 3 , session 2. (C) Two example neurons (highlighted in B) that changed their peak instantaneous firing rate (shaded areas, cartoon depictions of instantaneous firing rates) during two example replay events (displayed in B) in the same direction as their peak in-field firing rate difference (unshaded areas, actual ratemaps). The activity and properties of the example neurons are color coded throughout all the panels. (D) The peak in-field firing rate difference (Track1-Track2) significantly predicts the average peak instantaneous replay rate difference (Track1-Track2) in POST rest, but not PRE rest. Each data point is a neuron active on both tracks (PRE: $n=106$ cells, POST: $n=123$ cells).

First, we sought to investigate whether the contextually-driven place fields' rate modulation between tracks is conserved during offline replay. Using all place cells active on both tracks and stable across the whole run (fig.S1B and supplementary methods), we compared the place fields' peak in-field firing rate differences between tracks (Track1-Track2) to the difference (Track1Track2) in average peak instantaneous rate of those same cells during significant replay events (Fig. 1C). For replay events during POST, the difference in peak in-field firing rate between tracks was predictive of the difference in average peak instantaneous rates during replay events from both tracks [POST: $\beta=.04, \mathrm{~F}(1,121)=36.95, P<0.001, \mathrm{R} 2=.23$; Fig. 1D]. In other words, if a place field had a higher firing rate on Track 2 (compared to Track 1), it also tended to have a higher mean firing rate across all Track 2 replay events (compared to Track 1 events) during POST. This was not the case during PRE $[$ PRE: $\beta=.06, \mathrm{~F}(1,104)=0.75, P=0.39$, R2= .007; Fig. 1D]. This suggests that any contextually-driven rate modulation between two behavioral episodes is maintained during offline replay. This observation was consistent when using a more selective criteria of place field activity (e.g. place fields with higher minimum peak firing rate or place cells with a single field) (figs. S3 and S4A), as well as for alternative metrics for both replay firing rate (e.g. mean number of spikes per replay event and median replay rate) (fig. S4, B and C) and place fields firing rate (e.g. place field's area under the curve) (fig. S4D). Furthermore, as expected, selecting only cells that were classified as significantly rate modulated led to an even stronger effect (fig. S4E). 

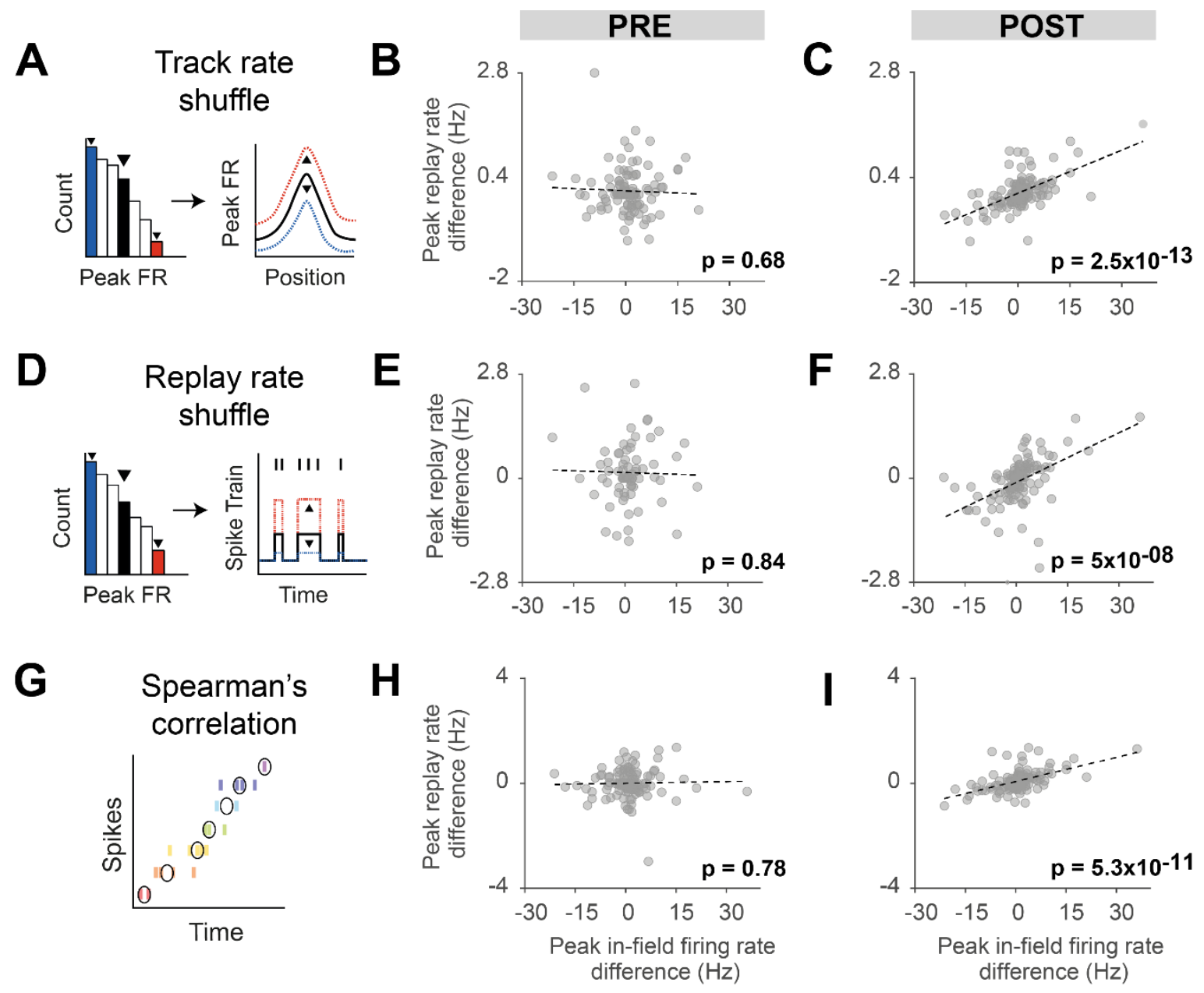

Fig. 2. The reinstatement of rate modulation during rest replay is observed using replay detection methods insensitive to firing rate. Three methods of replay detection that are insensitive to firing rate: (A) Track rate shuffle (PRE: $n=106$ cells, POST: $n=123$ cells), (D) Replay rate shuffle (PRE: $n=69$ cells, POST: $n=117$ cells). (A and D) Example of the original rate (right plot, in black) randomly scaled up (right plot, in red) or down (right plot, in blue), after drawing a random value from the rate distribution (left plot). (G) Spearman correlation, where black circles indicate median spike times (PRE: $n=111$ cells, POST: $n=123$ cells). (B, E, H) Regression of peak-in-field firing rate vs. peak replay rate difference (Track 1-Track 2) for PRE replay events with modified detection method: (B) Track rate shuffle, (E) Replay rate shuffle, (H) Spearman correlation. (C, F, I) Regression of peak-in-field firing rate vs. peak replay rate difference (Track 1-Track 2) for POST replay events with modified detection method (C) Track rate shuffle, (F) Replay rate shuffle, (I) Spearman correlation.

Replay detection methods generally detect the statistical significance of cells sequences based on templates derived from the behavioral episode, which are sensitive to the order of place field activation (during RUN and replay). However, because Bayesian decoding can also be influenced by firing rate, we next tested whether firing rate differences between tracks may bias the selection of the replay events used in the regression analysis. To remove the influence of firing rate in the replay event selection, we employed two independent types of shuffles before 
repeating the detection of replay events: 1) Track rate shuffle, which randomizes the overall firing rate of place fields within a track (Fig. 2, A to C) and 2) Replay rate shuffle, which randomizes the cell's firing rate during replay events (Fig. 2, D to F). For both shuffles, each place cell's firing rate was scaled based on a value randomly drawn from a distribution of firing rates obtained from the analyzed place cells on the track (for the Track rate shuffle) or during the replay events (for the Replay rate shuffle). These re-identified significant replay events were then used to repeat the regression analysis but using the original data (i.e. the original replay spike train and place fields, instead of the shuffled ones). For both shuffles, we observed a significant contextual rate modulation of replay, where track differences in the place fields' in-peak firing rate remained predictive of track differences in the average peak instantaneous rates during replay events in POST [POST: Track rate shuffle $P<.001$, Replay rate shuffle: $P<.001$; Fig. 2 , $\mathrm{C}$ and F], but not PRE [PRE: Track rate shuffle $P=.68$, Replay rate shuffle: $P=.84$; Fig. 2 , B and $E]$. As an additional control, we detected replay events using a rank order correlation method independent of Bayesian decoding (i.e. Spearman correlation), which relies only on comparing the median spike times across the place cells of a replay event compared to the sequential order of place field activity along the track (Fig. 2, G to I). Repeating the regression analysis based on the track differences in the place fields' in-peak firing rate against the replay events detected using a Spearman correlation still resulted in a significant contextual rate modulation of the replay events rate in POST, but not PRE $[\mathrm{PRE}: \beta=.002, \mathrm{~F}(1,111)=.08, P=.78, \mathrm{R} 2=.0007$, POST: $\beta=.03, F(1,121)=51.99, P<.001, \mathrm{R} 2=0.3$; Fig. $2, \mathrm{H}$ and I]. Overall, these results indicate that the contextual rate modulation observed during replay is not a result of a bias within the replay detection analysis (namely, Bayesian decoding). Importantly, we did not observe any significant track differences in place field or replay properties (fig. S5), including the place fields' peak in-field firing rate [two-sided two-sample KS test, $P=.62$, fig. S5B] and the replay average peak instantaneous rate [two-sided two-sample KS test, $P=.79$, fig. S5B], and confirmed that our regression analysis was not influenced by any intrinsic biases between tracks by remeasuring the statistical significance relative to a shuffled distribution (fig. S6).

The phenomenon of replay also occurs during awake quiescent states (e.g. while pausing on the track), and has been proposed to serve different functional roles, ranging from planning to memory storage $(9,16-19)$. We observed that during the behavioral episode on the tracks, contextual rate modulation was expressed across local awake replay events [RUN: $\beta=.05$, $\mathrm{F}(1,120)=197.02, P<.001, \mathrm{R} 2=.61 ; \mathrm{Fig} .3 \mathrm{~A}]$. Rate modulation during replay was even more closely aligned with behavior during RUN, compared to POST [RUN: F(1,120)=197.02, POST: $\mathrm{F}(1,121)=36.95$; Fig. 1D]. Place cells during RUN already showed evidence of contextual rate modulation during awake local replay events within the first 2 laps of experience [2 laps: $\beta=.06$, $\mathrm{F}(1,8)=5.91, P=.04, \mathrm{R} 2=.42$; Fig. 3B]. Furthermore, place cells expressing rate modulation during RUN replay events were also likely to show a similar direction of contextual rate modulation (e.g. Track $1>$ Track 2 ) during POST replay events $[\beta=.78, \mathrm{~F}(1,168)=95.36, P<$ $.001, \mathrm{R} 2=.36$; Fig. $3 \mathrm{C}]$, but not $\mathrm{PRE}[\beta=.02, \mathrm{~F}(1,143)=1.25, P=.2, \mathrm{R} 2=.008$, fig. S7]. Contextual rate modulation during replay was significantly expressed throughout the entire rest period during POST (at least 1 hour cumulatively, limited only by the maximum time period tested), with the strongest effect observed in the earliest portion of POST rest (Fig. 3D). 


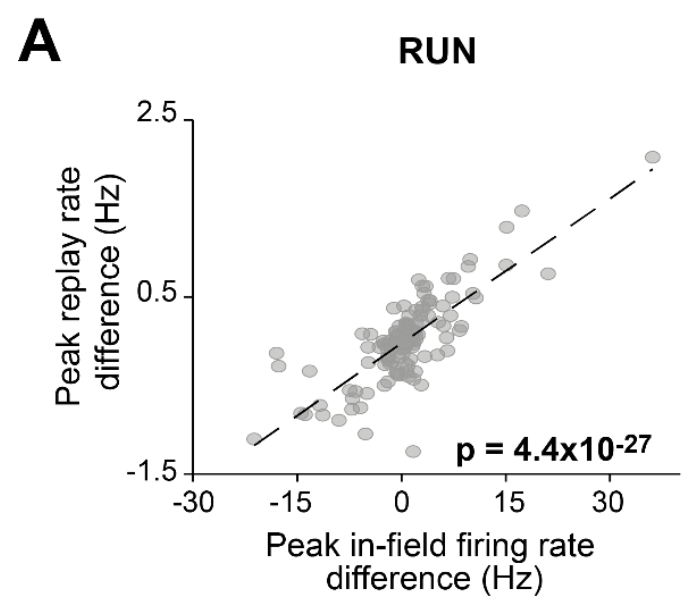

C

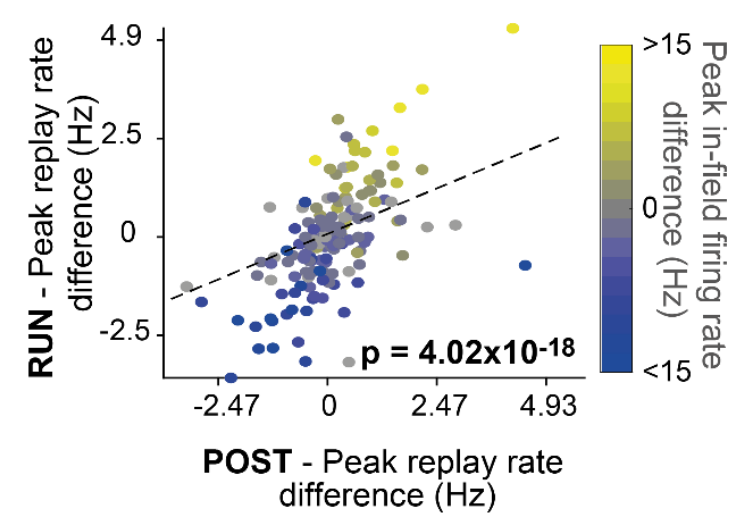

\section{B Emergence During RUN}

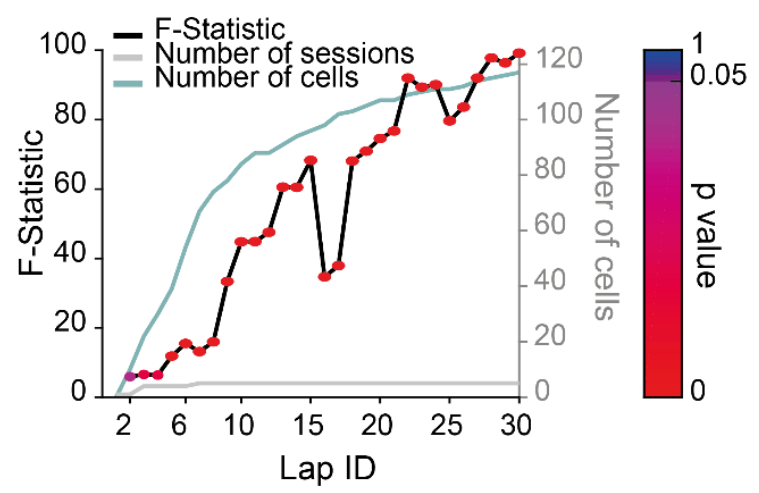

D Evolution over POST

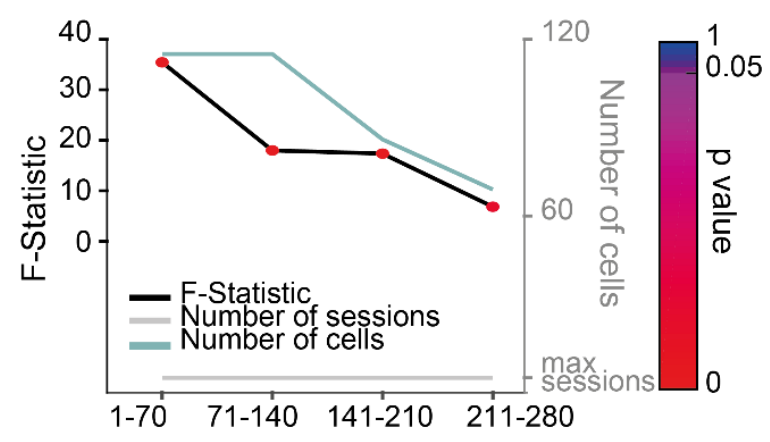

Event block (each session)

Fig. 3. Rate modulation during replay is observed from the beginning of RUN and continues throughout the entire RUN and POST epochs. (A) Regression of peak in-field firing rates against peak replay rates (track differences) for all local RUN replay events. (B) Lap by lap emergence of rate modulation for RUN local replay events. F-statistic of regression - peak in-field firing rate vs. peak replay rate (track difference) - for replay events occurring both prior to and including the specified lap (lap ID). The p-value of each regression is indicated by color. The number of cells and number of data sessions contributing in each regression are indicated with the green and grey line, respectively. (C) Peak replay rate difference for replay events (RUN vs. POST), color coded by peak in-field rate differences. (D) Temporal dynamics of rate modulation during POST. The regression's F-statistic (y-axis) and the corresponding p-value (marker color), analyzed in non-overlapping blocks of 70 consecutive replay events (per session). The number of cells and number of data sessions contributing in each regression are indicated with the green and grey line, respectively.

Our data suggest that both place (i.e. sequences) and rate information is embedded within hippocampal replay events. If this is the case, then the spatial context (i.e. track identity) associated with a replay event should be identifiable using either the place or rate representations alone. To address this question, we modified the current Bayesian framework such that it would rely on either or both place and rate information. For each decoded replay event, we measured the probabilistic bias towards one of the two tracks using the z-scored log odd (20), $\left(\log \frac{\sum \operatorname{prob}\left(\operatorname{Track}_{1}\right)}{\sum \operatorname{prob}\left(\operatorname{Track}_{2}\right)}\right)$. Thus, for a given replay event, this method summed the decoded posterior 
probability for each track, quantified the logarithmic ratio between the two tracks, and then zscored this ratio relative to a shuffled distribution (Ratemap track label shuffle, fig. S8 and supplementary methods). For example, a higher positive $\mathrm{z}$-scored log odd would indicate a greater probabilistic bias towards Track 1. Therefore, if the content of a replay event was detected for Track 1 (based on a statistically significant sequence), the corresponding z-scored log odd would reflect how well a place and/or rate representation could be used to correctly classify this replay event as Track 1 (rather than Track 2).

In particular, we used two measurements to assess the replay track identity classification performance when using either the rate and/or place representation (fig. S8 and supplementary methods): 1) the log odd difference between two tracks compared to a Replay track identity shuffled distribution and 2) the binary discriminability of a replay event's track identity using Receiver Operator Characteristic (ROC) curves, in which the area under the ROC curve (AUC) of 0.5 indicates chance discriminability (and an AUC of 1 indicates perfect discriminability). For both measurements, only replay events with a statistically significant replay sequence were used. When both place and rate information were unaltered, the $\mathrm{z}$-scored log odd difference and the track discriminability were highest for RUN $[\Delta(\mathrm{z}$-scored $\log$ odd $)=2.77, P<.001, \mathrm{AUC}=.98]$, significant but marginally lower for POST $[\Delta(\mathrm{z}$-scored $\log$ odd $)=1.29, P<.001$, AUC $=.80]$, but indistinguishable from the null distribution and at chance levels for $\operatorname{PRE}[\Delta(\mathrm{z}$-scored $\log$ odd $)=$ $.08, P=.22$, AUC= 0.52] (Fig. 4A and table S2).

We then proceeded to selectively remove either the firing rate or place information available to the Bayesian decoder and assess its ability to determine the track being replayed. First, we removed the firing rate information by both setting each place field's in-field peak firing rate to its average across both tracks and rescaling each cell's replay spike trains to its average firing rate across all replay events (Rate fixed manipulation). We found that place information alone was sufficient to correctly classify replay events to each track, although both the z-scored log odd difference and the AUC were slightly lower than when both place and firing rate information were available [AUC difference $<-0.1$ compared to the original for all task periods, Fig. 4, B and $\mathrm{F}$ to $\mathrm{H}$, and table $\mathrm{S} 2$ ]. 

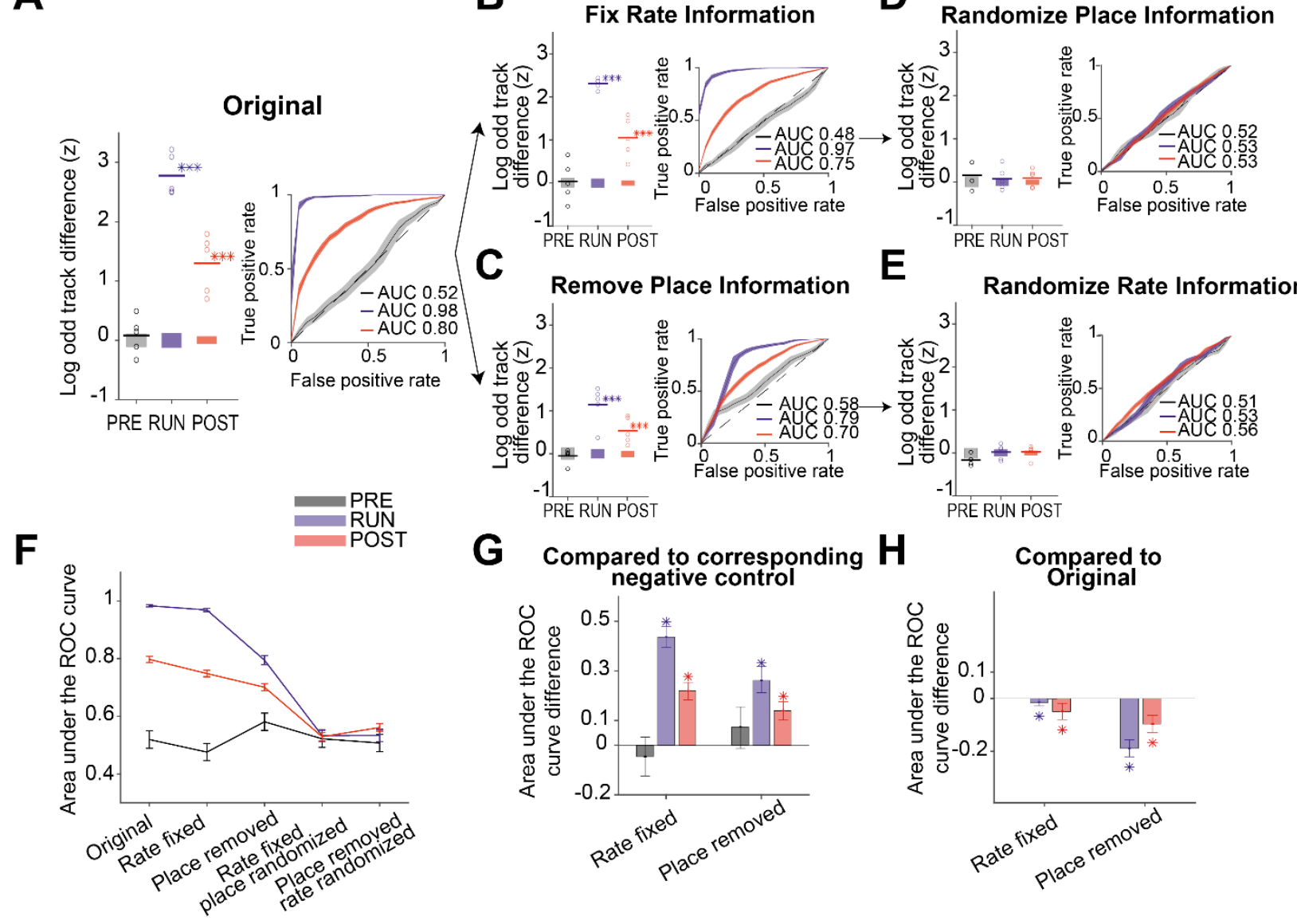

Fig. 4. Contextual information can be decoded from either place or rate representations during replay. (A to $\mathbf{E}$ ) The decoding performance for context (Track 1 or Track 2) for each manipulation, restricting the information (rate and/or place) available to the decoder. (Main plots) Mean z-scored log odd difference between tracks for replay during PRE (black), RUN (blue) and POST (red). Each data point is the mean of an individual session, while the horizontal line indicates the mean across all sessions $(* P<0.05, * * P<0.01, * * * P<0.001$, one-tailed). The filled box indicates the SEM from the Replay track identity shuffles. (Insets) ROC curves of Track1/Track2 binary discrimination for replay events during PRE, RUN and POST. The dashed line along the diagonal indicates chance performance ( $A U C=0.5$ ). The shaded region indicates the bootstrapped SE of the ROC curves. (A) Original (rate and place information intact), (B) Rate fixed manipulation (only place information available), (C) Place removed manipulation (only rate information available), (D) Rate fixed manipulation with place information additionally randomized (no rate or place information available), and (E) Place removed manipulation with rate information additionally randomized (no rate or place information available). (F) Summary of mean and SEM AUC under each manipulation condition, for PRE, RUN, and POST. (G) Mean AUC difference between each manipulation and the corresponding negative control. (H) Mean AUC difference between original and Rate fixed and Place removed manipulation. For $(\mathrm{G}$ and $\mathrm{H})$, asterisks $(*)$ indicate where the $95 \%$ confidence interval of the bootstrapped AUC difference distribution does not overlap with 0 (tables S3 and S4). Similar results were obtained using an alternative approach (Delong method) for measuring significance (tables S5 and S6, and supplementary methods). 
Next, we selectively removed place information without altering rate information, by expanding the size of both the position and time bins during the decoding: a single position bin spanning the entire track and a single time bin spanning the entire replay event (Place removed manipulation, Fig. 4, $\mathrm{C}$ and $\mathrm{F}$ to $\mathrm{H}$ ). This manipulation led to a significant $\log$ odd difference towards the correct track and a high track discriminability for RUN $[\Delta$ (z-scored log odds) $=1.14, P<.001$, $\mathrm{AUC}=.79]$, POST $[\Delta(\mathrm{z}$-scored $\log$ odds $)=.53, P<.001, \mathrm{AUC}=.70]$, but not PRE $(\Delta(\mathrm{z}$-scored $\log$ odds $)=-.04, P=0.63, \mathrm{AUC}=.58]$, supporting the finding that rate modulation is reinstated during replay and is experience-dependent. Finally, as a negative control for both manipulations, where rate and place representations were disrupted, each remaining non-manipulated representation (place or rate) was then randomized (Rate fixed place randomized manipulation and Place removed rate randomized manipulation, respectively). Removing both place and rate representations resulted in chance level discriminability for both conditions (Fig. 4, D to H, and table S2).

Here we have shown that contextually driven place and rate representations used by the hippocampus during behavior are reinstated during replay, with either representation being sufficient to successfully decode the context being replayed. Building on previous work supporting the contribution of firing rate $(10,13,21-23)$ in the encoding of behavioral episodes, we demonstrate that this phenomenon is experience-dependent, as rate modulation within replay was only observed during and after a behavioral episode, but not prior to it. While place cell sequences are generally stable across a behavioral episode within a single environment, in-field firing rates can still increase (or decrease) with minor contextual changes, a phenomenon referred to as rate remapping (12). Our results present a possible solution to this problem for the brain, as two marginally similar contexts producing identical sequential patterns during replay are still potentially separable due to their rate modulation differences.

Computational models of replay have commonly used modified synfire chains, sequentially connected neurons that are often embedded within distinct recurrent networks (24-26). While these models aim to replicate the sequential nature of replay, it remains to be seen whether they can also reinstate experience-dependent rate modulation, or if additional mechanisms may need to be considered. Compelling evidence suggests a key role for neocortical activity preceding a hippocampal replay event in determining its content (27-29). If cortical inputs to the hippocampus during behavior underlie rate modulation (30), a reinstatement of these same inputs prior to replay may be necessary for the subsequent rate modulation of hippocampal place fields during offline states.

\section{References and Notes}

1. J. O'Keefe, J. Dostrovsky, The hippocampus as a spatial map. Preliminary evidence from unit activity in the freely-moving rat. Brain Res. 34, 171-175 (1971).

2. M. A. Wilson, B. L. McNaughton, Reactivation of hippocampal ensemble memories during sleep. Science. 265, 676-9 (1994).

3. A. K. Lee, M. A. Wilson, Memory of sequential experience in the hippocampus during slow wave sleep. Neuron. 36, 1183-1194 (2002). 
4. G. Girardeau, K. Benchenane, S. I. Wiener, G. Buzsáki, M. B. Zugaro, Selective suppression of hippocampal ripples impairs spatial memory. Nat. Neurosci. 12, 1222-1223 (2009).

5. A. Fernández-Ruiz, A. Oliva, E. F. de Oliveira, F. Rocha-Almeida, D. Tingley, G. Buzsáki, Long-duration hippocampal sharp wave ripples improve memory. Science (80-. ). 364, 1082-1086 (2019).

6. R. Crowley, D. Bendor, A. H. Javadi, A review of neurobiological factors underlying the selective enhancement of memory at encoding, consolidation, and retrieval. Prog. Neurobiol. 179, 101615 (2019).

7. T. J. Davidson, F. Kloosterman, M. A. Wilson, Hippocampal Replay of Extended Experience. Neuron. 63, 497-507 (2009).

8. L. Genzel, G. Dragoi, L. Frank, K. Ganguly, L. De La Prida, B. Pfeiffer, E. Robertson, A consensus statement: defining terms for reactivation analysis. Philos. Trans. R. Soc. B Biol. Sci. 375 (2020), doi:10.1098/rstb.2020.0001.

9. D. J. Foster, M. A. Wilson, Reverse replay of behavioural sequences in hippocampal place cells during the awake state. Nature. 440, 680-683 (2006).

10. P. Ravassard, A. Kees, B. Willers, D. Ho, D. Aharoni, J. Cushman, Z. M. Aghajan, M. R. Mehta, Multisensory Control of Hippocampal Spatiotemporal Selectivity. Science (80-. ). 340, 1342-1346 (2013).

11. X. Zhao, Y. Wang, N. Spruston, J. C. Magee, Membrane potential dynamics underlying context-dependent sensory responses in the hippocampus. Nat. Neurosci., 1-11 (2020).

12. S. Leutgeb, J. K. Leutgeb, C. a Barnes, E. I. Moser, B. L. McNaughton, M. Moser, Independent Codes for Spatial and Episodic Memory in Hippocampal Neuronal Ensembles. Science (80-. ). 309, 619-624 (2005).

13. J. R. Huxter, N. Burgess, J. O'Keefe, Independent rate and temporal coding in hippocampal pyramidal cells. Nature. 425, 828-832 (2003).

14. A. V. Olypher, P. Lánský, A. A. Fenton, Properties of the extra-positional signal in hippocampal place cell discharge derived from the overdispersion in location-specific firing. Neuroscience. 111, 553-566 (2002).

15. J. D. Monaco, G. Rao, E. D. Roth, J. J. Knierim, Attentive scanning behavior drives onetrial potentiation of hippocampal place fields. Nat. Neurosci. 17, 725-731 (2014).

16. H. Xu, P. Baracskay, J. O’Neill, J. Csicsvari, Assembly Responses of Hippocampal CA1 Place Cells Predict Learned Behavior in Goal-Directed Spatial Tasks on the Radial Eight-Arm Maze. Neuron. 101, 119-132.e4 (2019).

17. B. E. Pfeiffer, D. J. Foster, Hippocampal place-cell sequences depict future paths to remembered goals. Nature. 497, 74-79 (2013).

18. A. K. Gillespie, D. A. Astudillo Maya, E. L. Denovellis, D. F. Liu, D. B. Kastner, M. E. Coulter, D. K. Roumis, U. T. Eden, L. M. Frank, Hippocampal replay reflects specific past experiences rather than a plan for subsequent choice. Neuron. 109, 1-15 (2021). 
19. K. Diba, G. Buzsáki, Forward and reverse hippocampal place-cell sequences during ripples. Nat. Neurosci. 10, 1241-1242 (2007).

20. A. Carey, Y. Tanaka, M. A. A. van der Meer, Reward revaluation biases hippocampal replay content away from the preferred outcome. Nat. Neurosci. 22, 1450-1459 (2019).

21. F. Savelli, J. J. Knierim, Origin and role of path integration in the cognitive representations of the hippocampus: computational insights into open questions. J. Exp. Biol. 222, 1-13 (2019).

22. C. D. Schwindel, Z. Navratilova, K. Ali, M. Tatsuno, B. L. McNaughton, Reactivation of rate remapping in CA3. J. Neurosci. 36, 9342-9350 (2016).

23. S. Takahashi, Episodic-like memory trace in awake replay of hippocampal place cell activity sequences. Elife. 4, 1-22 (2015).

24. M. Abeles, Role of the cortical neuron: integrator or coincidence detector? Isr. J. Med. Sci. 18, 83-92 (1982).

25. M. Diesmann, M.-O. Gewaltig, A. Aertsen, Stable propagation of synchronous spiking in cortical neural networks. Nat. 1999 4026761. 402, 529-533 (1999).

26. N. Chenkov, H. Sprekeler, R. Kempter, Memory replay in balanced recurrent networks. PLOS Comput. Biol. 13, e1005359 (2017).

27. D. Bendor, M. A. Wilson, Biasing the content of hippocampal replay during sleep. Nat. Neurosci. 15, 1439-1444 (2012).

28. P. A. Lewis, D. Bendor, How Targeted Memory Reactivation Promotes the Selective Strengthening of Memories in Sleep. Curr. Biol. 29 (2019), pp. R906-R912.

29. G. Rothschild, E. Eban, L. M. Frank, SI - A cortical - hippocampal - cortical loop of information processing during memory consolidation. Nat. Neurosci., 1-12 (2016).

30. C. Rennó-Costa, A. B. L. Tort, Place and grid cells in a loop: implications for memory function and spatial coding. J. Neurosci. 37, 3490-16 (2017).

31. K. Zhang, I. Ginzburg, B. L. McNaughton, T. J. Sejnowski, Interpreting neuronal population activity by reconstruction: Unified framework with application to hippocampal place cells. J. Neurophysiol. 79, 1017-1044 (1998).

32. X. Sun, W. Xu, Fast implementation of DeLong's algorithm for comparing the areas under correlated receiver operating characteristic curves. IEEE Signal Process. Lett. 21, 13891393 (2014).

Acknowledgments: We thank Yu Qian, Sophie Renaudineau and Julieta Campi for technical assistance; members of the Bendor Lab for valuable discussion; and Aman Saleem and Caswell Barry for their helpful comments on the manuscript. Rat schematic in Fig. 1A adapted with permission from SciDraw.io (doi.org/10.5281/zenodo.3926277, doi.org/10.5281/zenodo.392637, https://creativecommons.org/licenses/by/4.0). 


\section{Funding:}

Biotechnology and Biological Sciences Research Council scholarship (BB/M009513/1) (MTir)

European Research Council starter grant (CHIME) (DB)

Human Frontiers Science Program Young Investigator Award (RGY0067/2016) (DB)

Biotechnology and Biological Sciences Research Council Research grant (BB/T005475/1) (DB)

The Titan Xp used for this research was donated by the NVIDIA Corporation

\section{Author contributions:}

Experimental design: MTir, MHG, DB

Methodology: MTir, MHG, LK

Data collection: MTir, MHG

Data analysis: MTir, MHG, MTak

Writing - initial draft: MTir, MHG, MTak, DB

Writing - editing: MTir, MHG, MTak, LK, DB

Order of co-first authorship was determined by coin-flip. Both MTir and MHG contributed equally and agree to reserve the right to list their name first in their $\mathrm{CV}$.

Competing interests: The authors declare that they have no competing interests.

Data and materials availability: All data and code used in this manuscript are available from the authors upon reasonable request. 


\section{Materials and Methods}

Animals

Three male Lister-Hooded rats (350-450g) were implanted with a microdrive with 24 independently moveable tetrodes. Prior to surgery, rats were kept at $90 \%$ of their free-feeding weight and housed in pairs on a 12-hour light/dark cycle, with 1 hour of simulated dusk/dawn. All experimental procedures and postoperative care were approved and carried out in accordance with the UK Home Office, subject to the restrictions and provisions contained within the Animal (Scientific Procedures) Act of 1986.

\section{Surgery}

Animals were deeply anaesthetized under isoflurane anesthesia (1.5-3\% at $2 \mathrm{~L} / \mathrm{min})$ and implanted with a custom-made microdrive array carrying 24 independently moveable tetrodes (modified from microdrive first published by (31)). Each tetrode consisted of a twisted bundle of four tungsten microwires ( $12 \mu \mathrm{m}$ diameter, Tungsten $99.95 \% \mathrm{CS}$, California Fine Wire), goldplated to reduce impedance to $<200 \mathrm{k} \Omega$. One rat was implanted with a dual-hippocampal microdrive targeting both dorsal hippocampal CA1 areas (AP: -3.48mm, ML: +/-2.4mm from Bregma), each output carrying 12 tetrodes. The two remaining rats were implanted with a microdrive targeting the right dorsal hippocampal CA1 area (AP: $3.72 \mathrm{~mm}$, ML: $2.5 \mathrm{~mm}$ from Bregma) and the left primary visual cortex (AP: $-5.76 \mathrm{~mm}$, ML: $-3.8 \mathrm{~mm}$ from Bregma), using 16 and 8 tetrodes respectively. After surgery, animals were housed individually and allowed to recover with food and water ad libitum for a week before returning to being kept at $90 \%$ of their free-feeding weight.

\section{Experimental design}

A given recording session started with a 1-hour rest period in which the rats were placed in a quiet, remote location (rest pot), to which they had been previously habituated. The rest pot consisted of a black circular enclosure of $20 \mathrm{~cm}$ of diameter, surrounded by a $50 \mathrm{~cm}$ tall black plastic sheet that isolated them from the surroundings. Following the rest period, the rats were exposed to two novel $2 \mathrm{~m}$ linear tracks in which they were allowed to run back and forth for 15 min, except for one session in which the animal ran for $30 \mathrm{~min}$ in the second track. Liquid reward was dispensed at each end of the track $(0.1 \mathrm{~mL}$ chocolate flavored soy milk) to encourage the animals to traverse the entirety of the track. In all except one session, the exposures to the two tracks were separated by a $10 \mathrm{~min}$ rest period in the rest pot. The recording session finished with a final 2-hours rest period inside the rest pot.

To simulate novel environments, the shape of the tracks was changed between recording sessions and their surfaces covered with different textured fabrics. In each session, the room was surrounded by black curtains with different high contrast visual cues. The tracks were separated using view-obstructing dividers.

\section{Spike detection and unit isolation}

Spiking data was sorted using the semi-automatic clustering software KlustaKwik 2.0 (K.Harris, http://klustakwik.sourceforge.net/) and then manually curated with either Phy-GUI (https://github.com/kwikteam/phy) or Klustaviewa (https://github.com/klusta-team/klustaviewa). Putative single units were discriminated based on the spike waveform, a clean inter-spike 
interval, and their stability across the recording session. The rest of the clustered activity was classified in either multi-unit activity or noise.

\section{$\underline{\text { LFP analysis }}$}

The power spectral density (PSD) of the LFP was calculated using Welch's method (pwelch, MATLAB) to identify the channels with higher power for theta (4-12Hz) and ripple $(125-300 \mathrm{~Hz})$ oscillations, as well as the channel with the largest difference in normalized theta to ripple power. The LFP of selected channels was next downsampled from $30 \mathrm{kHz}$ to $1 \mathrm{kHz}$ and band-passed filtered (MATLAB command filtfilt). The instantaneous phases were estimated using Hilbert transform.

\section{Criteria for Place cell selection}

Putative principal cells were identified by selecting units with a Half Width Half Max (HWHM) larger than $500 \mu$ s and mean firing rate $<5 \mathrm{~Hz}$ across the entire recording session. For place cell classification, spike trains were speed-filtered to only include the spiking activity between $4 \mathrm{~cm} / \mathrm{s}$ and $50 \mathrm{~cm} / \mathrm{s}$. A principal cell was classified as a place cell according to the following criteria: 1) the minimum peak firing rate was $>1 \mathrm{~Hz}$ in the unsmoothed ratemap and 2) a stable spiking activity across the first half and second half of the exploration on the track. Only place cells satisfying these two criteria for each linear track independently were included in the regression analysis (Figs. 1, 2 and 3) and log odd analysis (Fig. 4).

To generate firing ratemaps (the spike histogram divided by the total dwell time at each position bin), the position data was discretized in $2 \mathrm{~cm}$ bins for visualization and plotting, and $10 \mathrm{~cm}$ bins for Bayesian decoding. Only raw (unsmoothed) ratemaps were used for all Bayesian decoding analyses.

\section{$\underline{\text { Population vector analysis (PPV) }}$}

A population vector analysis was used to assess the ratemap stability for each track as well as the degree of between track remapping (12). For each session, the linearized ratemaps of all recorded pyramidal cells with a firing rate $>1 \mathrm{~Hz}$ were stacked into a 20 position bins $\mathrm{x} \mathrm{N}$ cells matrix for each track. To assess remapping between tracks, the ratemaps computed from the entire track experience were compared, while to assess ratemap stability within a track, a ratemap was computed for both the first and second half of the behavioral episode. The population vector of rates at each position bin was then correlated with its counterpart vector: either on the other track or during the other half of the experience. The mean Pearson correlation value was first averaged across position bins and then across sessions.

\section{Bayesian Decoding}

A naïve Bayesian decoding algorithm was applied to reconstruct the animal's spatial trajectory during both behavior and replay events from CA1 hippocampal spiking activity (7):

$$
P(x \mid n)=C P(x)\left(\prod_{i=1}^{N} f_{i}(x)^{n_{i}}\right) \exp \left(-\tau \sum_{i=1}^{N} f_{i}(x)\right)
$$

where $\mathrm{P}$ is the probability of the animal being at a specific position given the observed spiking activity, $C$ is a normalization constant, $x$ is the subject's position, $f_{i}(x)$ is the firing rate of the $i^{\text {th }}$ 
place field at a given location $\mathrm{x}$, and $\mathrm{n}$ is the number of spikes in the time window $\tau$. The normalization constant was re-defined as the summed posterior probabilities across both tracks. The maximum probability of the subject's position was decoded using $250 \mathrm{~ms}$ and $20 \mathrm{~ms}$ time bin during behavior and replay, respectively.

The decoding error was defined as the differences between the real location of the animal and the estimated position with maximum-likelihood.

\section{Candidate replay event detection}

Candidate replay events were detected based on MUA and ripple power. MUA was first smoothed with a Gaussian Kernel (sigma $=5 \mathrm{~ms}$ ) and binned into $1 \mathrm{~ms}$ steps. Only MUA bursts with a maximum duration of $300 \mathrm{~ms}$ and z-scored activity over 3 were selected. Ripples LFP signal was smoothed with a $0.1 \mathrm{~s}$ moving average filter and a threshold over 3 was set for the $\mathrm{z}$ scored ripple power.

Candidate replay events passing both thresholds were next speed-filtered (above $5 \mathrm{~cm} / \mathrm{s}$ ), and discarded if their sequence had less than 5 different units active or if their duration was below $100 \mathrm{~ms}$ or over $750 \mathrm{~ms}$ (thus, candidate events had at least 5 consecutive $20 \mathrm{~ms}$ bins). Events detected within $50 \mathrm{~ms}$ were combined. In an effort to optimize detection of replay events and avoid a minority of events that were discarded due to noisy probability decoding at the beginning or the end of the event, candidate replay events were split in half. To do so, the minimum MUA activity in the middle third of the candidate replay event was used to determine a natural midpoint to split the event in two segments. Both segments were decoded and tested for significance independently following the same procedure as the 'intact' candidate replay events (i.e., same criteria including minimum duration, etc.), for the exception of an adjusted p-value threshold $(P<0.025)$.

Replay events were classified as rest or awake local replay. Awake replay was defined as replay events occurring while the animals were running on either of the tracks and classified as local if the content of the replay event reflected the current track on which the animal was running. Replay events detected during sleep or rest periods within the rest pot were classified as rest replay events.

\section{Replay events scoring and significance}

Replay events were scored using weighted correlation between decoded posterior probabilities across position and time.

Weighted mean:

Weighted covariance:

$$
m(x ; \text { prob })=\frac{\sum_{i=1}^{M} \sum_{j=1}^{N} \operatorname{prob}_{i j} x_{i}}{\sum_{i=1}^{M} \sum_{j=1}^{N} \operatorname{prob}_{i j}}
$$

$$
\operatorname{cov}(x, t ; \operatorname{prob})=\frac{\sum_{i=1}^{M} \sum_{j=1}^{N} \operatorname{prob}_{i j}\left(x_{i}-m(x ; \operatorname{prob})\right)\left(t_{j}-m(y ; \operatorname{prob})\right)}{\sum_{i=1}^{M} \sum_{j=1}^{N} \operatorname{prob}_{i j}}
$$

Weighted correlation:

$$
\operatorname{corr}(x, t ; \operatorname{prob})=\frac{\operatorname{cov}(x, t ; \text { prob })}{\sqrt{\operatorname{cov}(x, x ; \operatorname{prob}) \operatorname{cov}(t, t ; p r o b)}}
$$


Where $x_{i}$ is the $\mathrm{i}^{\text {th }}$ position bin, $t_{j}$ is the $\mathrm{j}^{\text {th }}$ time bin and prob $_{i j}$ is the probability at the position bin $i$ and time bin $j$.

The statistical significance of the weighted correlation for each candidate replay event was assessed by comparison with three different 1000 shuffle distributions:

1) Spike train circular shift, in which the spike count vectors for each cell were independently circularly shifted in time within each replay event, prior to decoding.

2) Place field shift, in which each ratemap was circularly shifted in space by a random amount of position bins prior to decoding.

3) Circular shift of position, in which posterior probability vectors for each time bin were independently circularly shifted by a random amount.

If the score of the candidate event was greater than the 95th percentile of the distribution for all three shuffles then the event was considered to be significant. In a few occasions, replay events were found to be significant for both tracks. Those events were assigned to one of the tracks by computing the "Bayesian bias" score for each track. Each score was calculated as the sum of the posterior probability matrix for one track, normalized by the total sum across tracks. To assign the replay event to a specific track the Bayesian bias score had to be greater than $60 \%$, otherwise the event was discarded.

\section{$\underline{\text { Reinstatement of rate modulation analysis }}$}

Linear regression was used to measure the reinstatement of rate modulation between place fields on the track and replay events (MATLAB function fitlm). For each place cell active on both tracks, rate modulation on the track was measured by calculating 1) the difference in peak in-field firing rate across tracks, and 2) the difference in the area under the curve (AUC; MATLAB function trapz). Place fields with a peak in-field firing rate $<1 \mathrm{~Hz}$ on either track were excluded. As a control, a variation of the analysis was done by excluding all cells with multiple place fields on the same track. Multiple peaks for a single cell were considered to be different place fields when their peak firing rate was $>1 \mathrm{~Hz}$, each place field width was over 2 spatial bins $(20 \mathrm{~cm})$, and the distance between the peaks was of 4 spatial bins $(40 \mathrm{~cm})$.

Rate modulation during replay events was calculated as the change in each cell's firing activity between the replay events encoding for each track. Included cells had to participate in at least one replay event for each track. The firing rate change was measured as 1) the difference in the average peak instantaneous rate across tracks, 2) the difference in the median rate across replay events, and 3) the difference in the mean number of spikes across replay events.

To obtain the peak instantaneous rate of each event, each cell's spike train was binned into $1 \mathrm{~ms}$ steps, filtered with a $100 \mathrm{~ms}$ long Gaussian window with $\sigma=20 \mathrm{~ms}$ (MATLAB command filter), and the peak amplitude of the resulting signal measured.

\section{Controls for replay detection analysis}

Two types of shuffles were applied to the data before repeating the detection of replay events using Bayesian decoding: a Track rate shuffle and Replay rate shuffle. The Track rate shuffle consisted in the randomization of the firing rate of the place fields within a track, while the Replay rate shuffle randomized the cells' firing rate during the replay events. For both shuffles, each place cell's firing rate was scaled based on a value randomly drawn from a 
distribution of firing rates obtained from the analyzed place cells on the track (for the Track rate shuffle) or during the replay events (for the Replay rate shuffle). The shuffled data was then used to repeat the detection of replay events, and the newly identified significant replay events were used in a linear regression comparing their original spike content (instead of the shuffled one) against the original differences in the peak in-field rate.

\section{Remapping classification}

A bootstrapping procedure was used to identify the rate and place modulation properties of each cell. Modulation was defined when the track difference in a place field's specific parameter (e.g. peak in-field firing rate or peak location) exceeded the intrinsic variability of such parameter on a single track. The distribution of each parameter was computed for both tracks by calculating firing ratemaps from a randomly sampled $\mathrm{N}$ out of $\mathrm{N}$ laps (1000 iterations, with replacement). The median value of a track distribution was then compared to the $5^{\text {th }} / 95^{\text {th }}$ percentiles of the other track, and vice versa. If either median exceeded either percentile, the cell was classified as being modulated between tracks for that parameter.

\section{Sequenceless Bayesian Decoding}

The sequenceless Bayesian decoding was modified from Carey et al. (2019) (20). Only cells with stable place fields on the track in the first and second half of the behavioral episode (peak in-field firing rate $>1 \mathrm{~Hz}$ ) were included in our sequenceless decoding analysis.

Before Bayesian decoding, ratemaps for Track 1 and Track 2 were concatenated as a single matrix [nCell x (nPosition(T1) + nPositions(T2))]. Unless otherwise mentioned, 20ms time bins and $10 \mathrm{~cm}$ position bins were used for decoding. After decoding, the posterior probabilities for each time bin were normalized across all position bins from both tracks to sum to 1 . Then, the summed posterior probability across all time bins within each replay event was computed, and the probabilistic bias towards Track 1 or Track 2 for each replay event was quantified as $\log \left(\frac{\sum \operatorname{Prob}\left(\operatorname{Track}_{1}\right)}{\sum \operatorname{Prob}\left(\operatorname{Track}_{2}\right)}\right)(\log$ odds$)$, where $\sum \operatorname{Prob}\left(\operatorname{Track}_{1}\right)$ and $\sum \operatorname{Prob}\left(\operatorname{Track}_{2}\right)$ referred to the summed posterior probabilities for Track 1 and Track 2 , respectively. To remove any intrinsic probabilistic bias, each raw log odd calculation was then z-scored relative to a shuffled log odd distribution (Ratemap track label shuffle), obtained by randomly permuting each cell's Track 1 and Track 2 ratemaps 1000 times for each replay event. The permutation was done by either keeping a cell's ratemap unaltered or performing a between track swap.

To test the decoding when only using place or rate information, the original data were manipulated as the following:

- Rate fixed manipulation: Rate information was fixed both by rescaling each cell's peak infield firing rate to the mean value across both tracks, and by rescaling its spike count in each replay event to the average firing rate across all replay events.

- Place removed manipulation: The place (and sequence) information was removed by decoding with only a single position bin, which encompassed the entire track (mean firing rate on each track when speed is above $5 \mathrm{~cm} / \mathrm{s}$ ) and a single time bin, which spanned the entire replay event.

Two negative controls were used to disrupt both rate and place information: 
- Rate fixed place randomized manipulation: After the Rate fixed manipulation, place information was randomized using a cell ID shuffle in which all cells were randomly reassigned to a different cell's ratemap for each replay event.

- Place removed rate randomized manipulation: After Place removed manipulation, rate information was randomized by scaling the firing rate on each track based on a value randomly drawn from a distribution of firing rates obtained from the analyzed place cells on the track.

\section{$\underline{\text { ROC analysis }}$}

The 'ground truth' track identity for each replay event was assigned based on the sequenceness of the replay, which was determined by comparing the event's weighted correlation score to three shuffled distributions. The binary discriminability of a replay event's identity (Track 1 or Track 2) during PRE, POST or RUN was quantified using the Receiver Operative Characteristic (ROC) curve. To ROC curve was constructed based on a range of true and false positive rates obtained by systematically shifting the discrimination threshold along the z-scored log odd distributions, for both Track 1 and Track 2 replay events. A trapezoidal approximation was used to estimate the area under each ROC curve (MATLAB function perfcurve for both constructing ROC curve and estimating area under the curve). The bootstrapped distributions of ROC curves and the area under the corresponding ROC curves were produced by resampling with replacement 1000 times.

\section{$\underline{\text { Statistics }}$}

\section{Z-scored Log Odd significance}

The statistical significance of the z-scored log odd difference between Track 1 and Track 2 was determined by comparison with a shuffled distribution in which replay events were resampled with replacement 1000 times and their track identities were randomly assigned. The single z-scores were computed relative to the shuffled distributions, after which the associated one tailed p-value were calculated. The log odd difference was considered significantly different from the shuffled distribution at the significance level of 0.05 .

\section{Bootstrapping for ROC significance}

To determine if rate and/or place representation manipulations significantly changed the area under the curve (AUC) of the ROC curves, we calculated the confidence interval for the difference between each condition's bootstrapped AUC distribution and that of the original data. This was repeated in an additional analysis by replacing the original data by one of the negative controls (Rate fixed place randomized manipulation and Place removed rate randomized manipulation). The mean difference between two bootstrapped AUC distributions were only considered statistically significant when the $95 \%$ confidence interval did not overlap with 0 .

\section{Delong Test for ROC}

To determine if rate and/or place manipulations statistically significantly changed the AUC of ROC curves within the same behavioral epochs, the AUC of both manipulations (i.e. Rate fixed or Place removed) were compared with the AUC of the original data or their corresponding negative controls (i.e. Rate fixed Vs Rate fixed place randomized and Place removed Vs Place removed rate randomized). The DeLong Test (32) was employed to perform pair-wise comparisons between two ROC curves. The DeLong variance-covariance matrix for 
two ROC curves was computed using MATLAB-based algorithm [https://github.com/PamixSun/DeLongUI] developed by Sun and Xu (2014) (32). A z-score was calculated based on the following equation:

$$
z=\frac{\left|A U C_{A}-A U C_{B}\right|}{\sqrt{\operatorname{var}(A)+\operatorname{var}(B)-2 \operatorname{cov}(A, B)}}
$$

where AUC referred to the area under the ROC curve, $\operatorname{var}(\mathrm{X})$ referred to the variance of ROC curves from the 1000 bootstrapped distribution, and $\operatorname{cov}(\mathrm{X})$ referred to the covariance of ROC curves from the 1000 bootstrapped distribution.

The z-score for each pair of comparisons was subsequently used to calculate the two-tailed pvalue. Two AUC distributions were considered significantly different when $P \leq 0.05$.

Code Availability

All analysis was carried out using MATLAB (Mathworks, Natick MA) and all code is available from the authors upon reasonable request. 
A

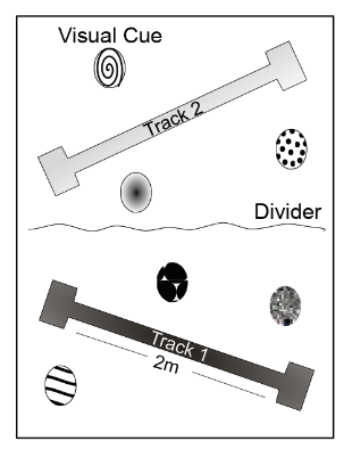

B

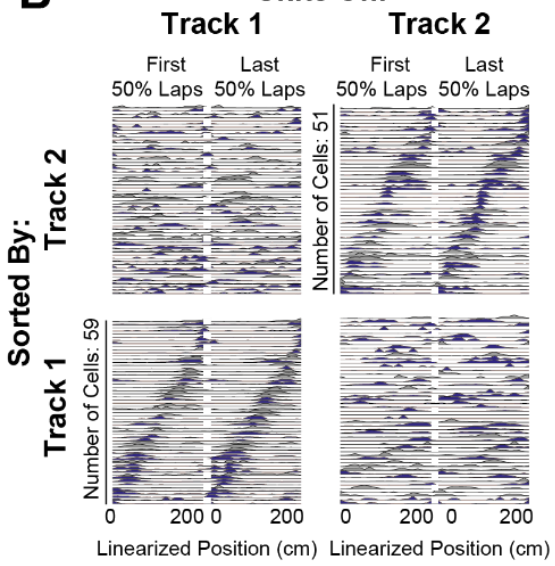

C

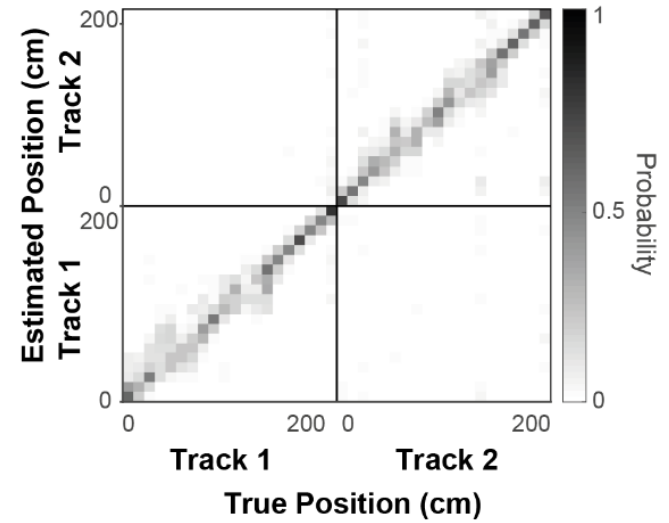

Fig. S1. Experimental setup, remapping and field stability. (A) Layout of the recording room - two linear tracks, each surrounded by visual cues and visually occluded from each other. (B) Ratemaps for Track 1 (left column) and Track 2 (right column) in an example session (rat 3, session 2) computed for the first 50\% of laps (left half) and last 50\% of laps (right half) and sorted by the ordered position of place fields on Track 1 (bottom) and Track 2 (top). (C) Confusion matrix for decoded position (posterior probability normalized over both tracks) for an example session (rat 3, session 2). Distribution of peak posterior probabilities (yaxis) is plotted at each True position during the behavioral episode (x-axis). 


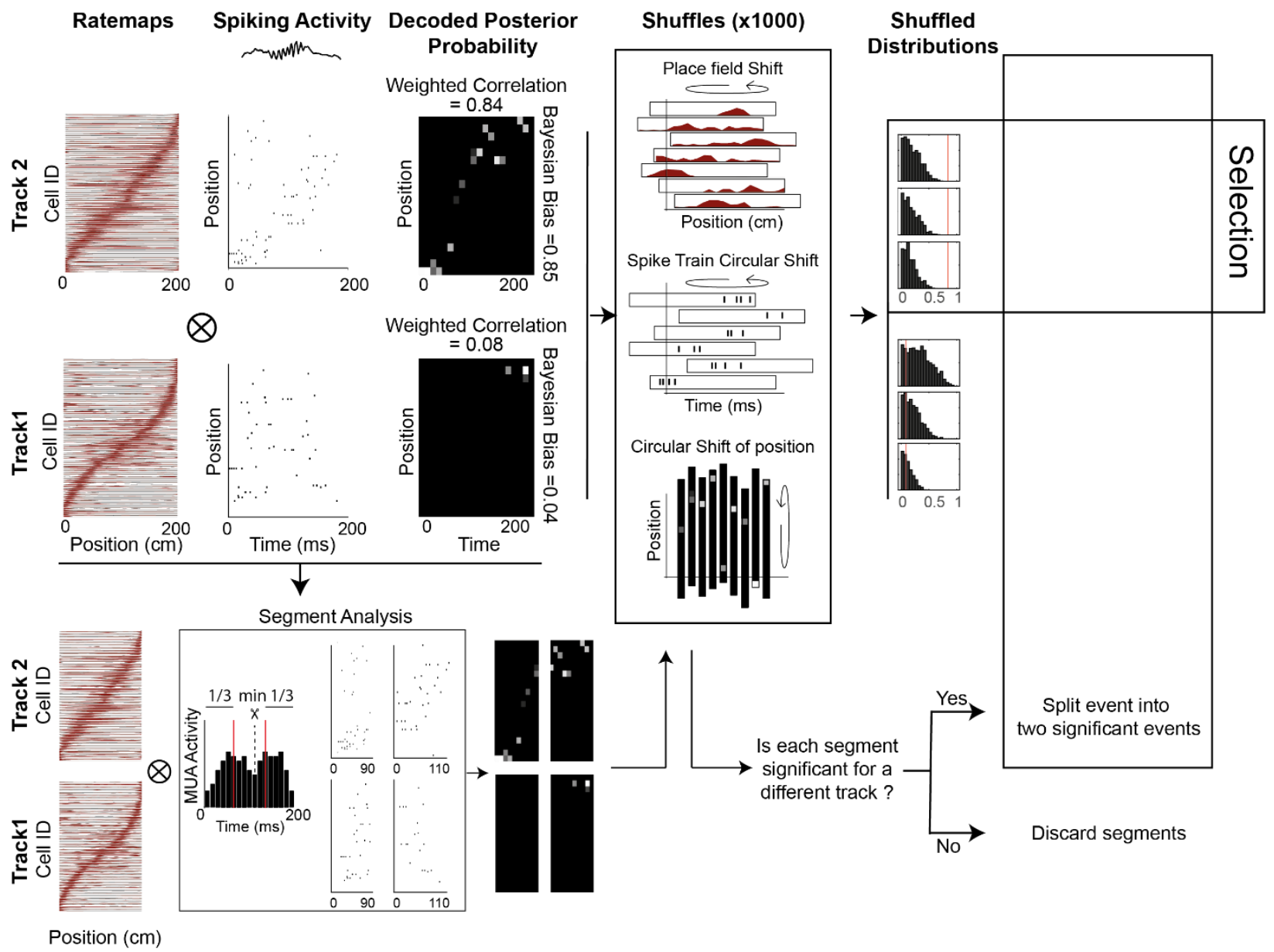

Fig. S2. Analysis pipeline for detection of significant replay events. Detected replay spike trains were decoded using a Bayesian framework. Each track's ratemaps obtained during RUN were used to calculate the decoded posterior probabilities for each replay sequence (top left). In addition, all replay events were split into two shorter events based on the minimum in the MUA during the middle third of the replay event (bottom left, Segment analysis). For each replay event, weighted correlations were calculated on decoded posterior probability from both the whole replay sequence and each segment, and the three scores were compared to three different shuffles (Place field shift, Spike train circular shift, and Circular shift of position, 1000 shuffles performed for each type). Only events with weighted correlation scores higher than $95 \%$ for every shuffle distribution ( $97.5 \%$ for split replay events) were considered statistically significant. 


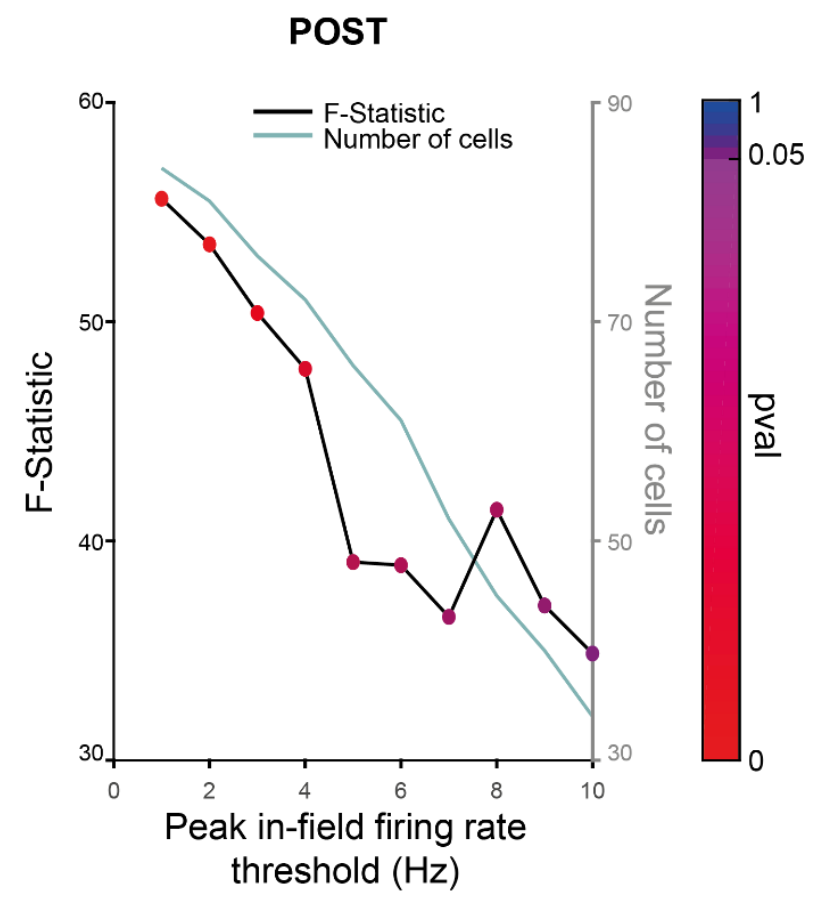

Fig. S3. Significance of regression analysis using a stricter criterion of place cell activity. F-statistic of regression - peak in-field firing rate vs. peak replay rate (track difference) - for replay events occurring during POST rest, as the criterion for place cell activity (minimum peak in-field firing rate) is progressively increased from $1 \mathrm{~Hz}$ to $10 \mathrm{~Hz}$. All regressions are statistically significant (up to and including using a threshold of $10 \mathrm{spk} / \mathrm{s}$ ). The p-value of each regression is indicated by color. The number of cells used in each regression is indicated by the green line. 


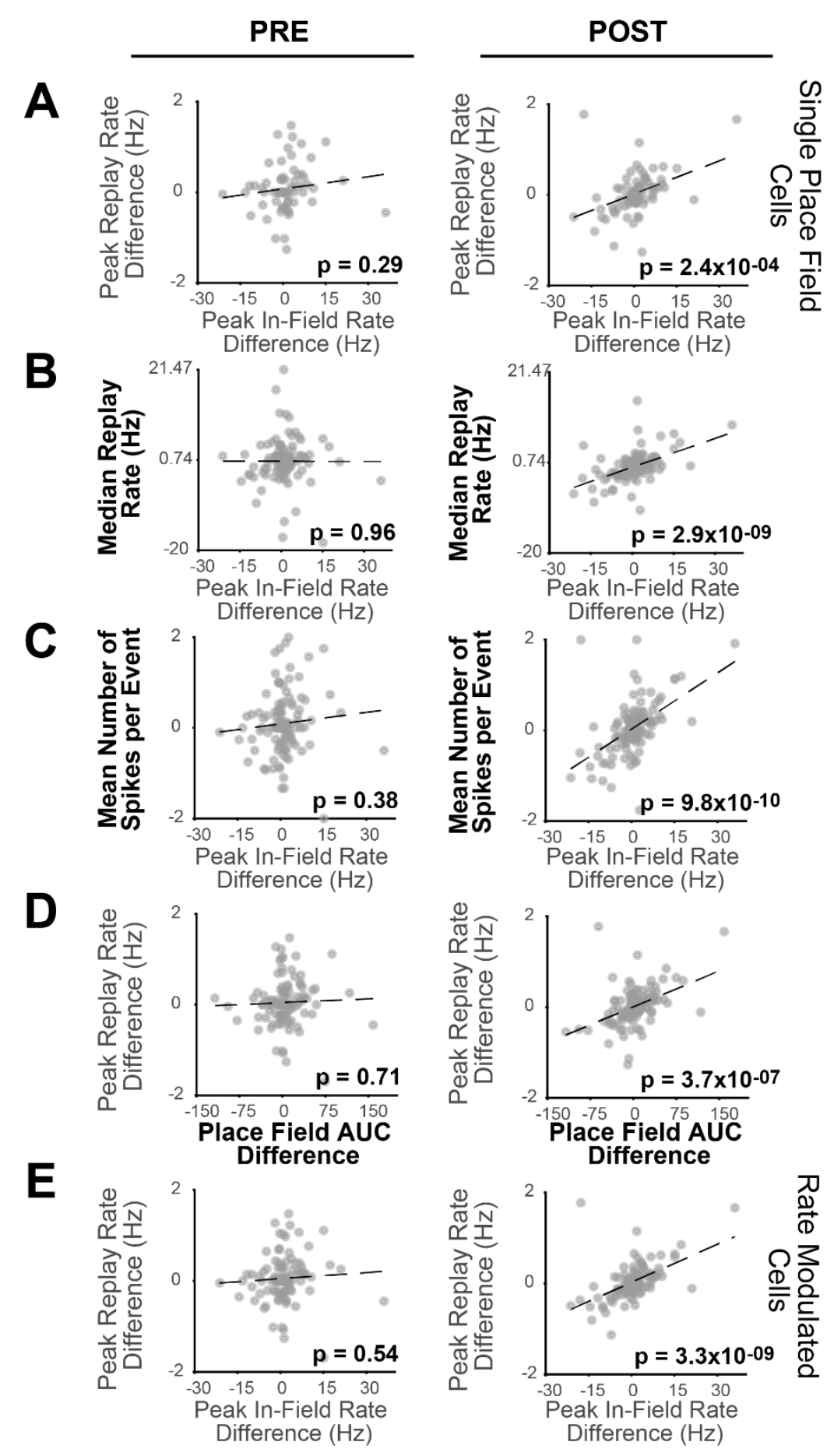

Fig. S4. Significance of regression analysis with alternative place field and replay rate metrics, and stricter criteria for cell selection. F-statistic of regression - peak in-field firing rate vs. peak replay rate (track difference) - for replay events occurring during PRE (left column) and POST (right column) using alternative metrics: (A) Place field area under the curve (new x-axis, PRE: $\mathrm{n}=106$ cells, POST: $\mathrm{n}=123$ cells), (B) Median replay rate (new y-axis, PRE: $\mathrm{n}=106$ cells, POST: $\mathrm{n}=123$ cells), and (C) Mean number of spike per event (new y-axis, PRE: $\mathrm{n}=106$ cells, POST: $\mathrm{n}=123$ cells). And using new criteria for cell selection: (D) Rate modulated cells (PRE: $\mathrm{n}=97$ cells, POST: $\mathrm{n}=112$ cells) and (E) Single place field cells (PRE: $\mathrm{n}=64$ cells, POST: $\mathrm{n}=77$ cells). 


\section{A}
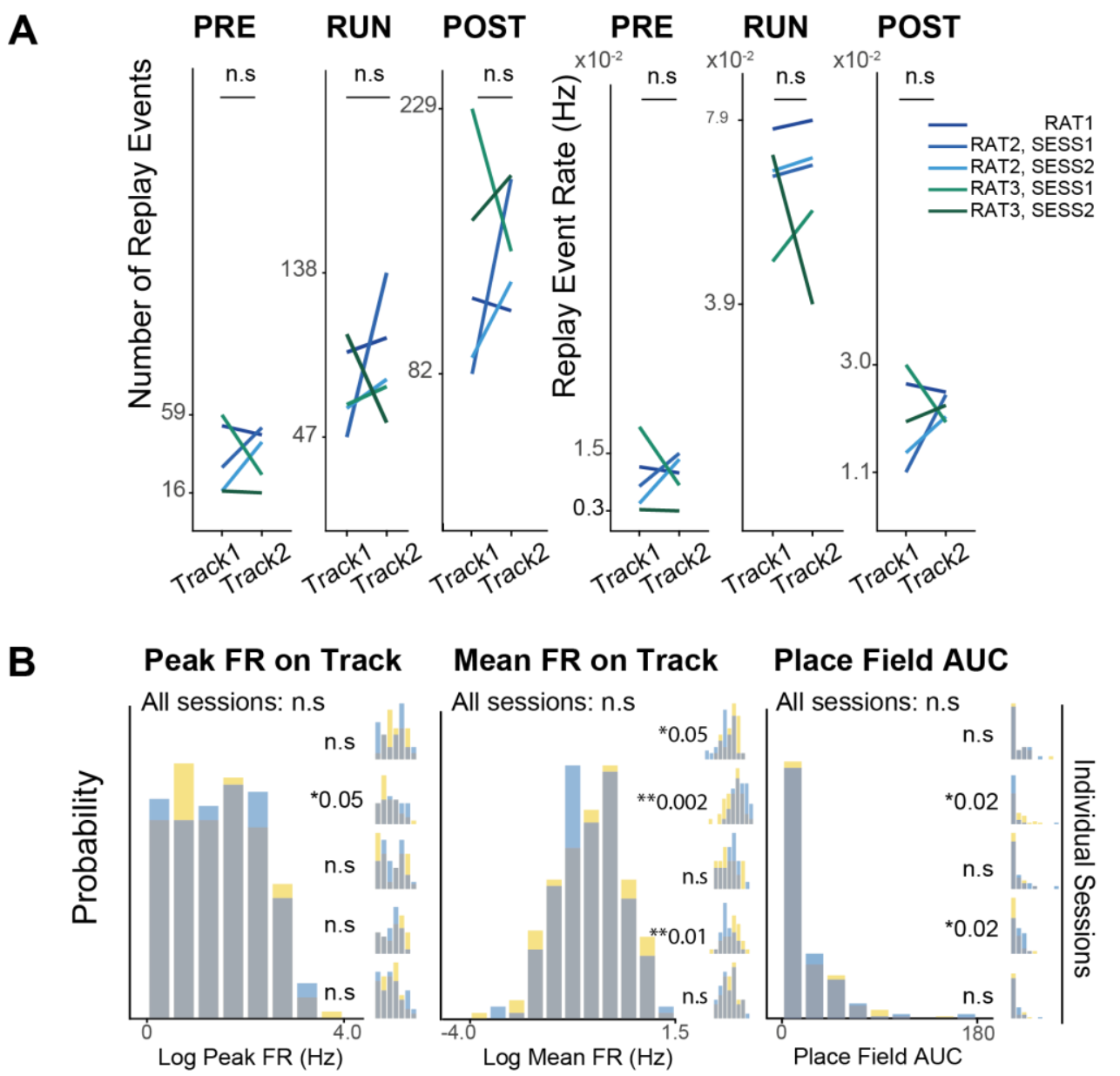

Mean FR on Track

Place Field AUC
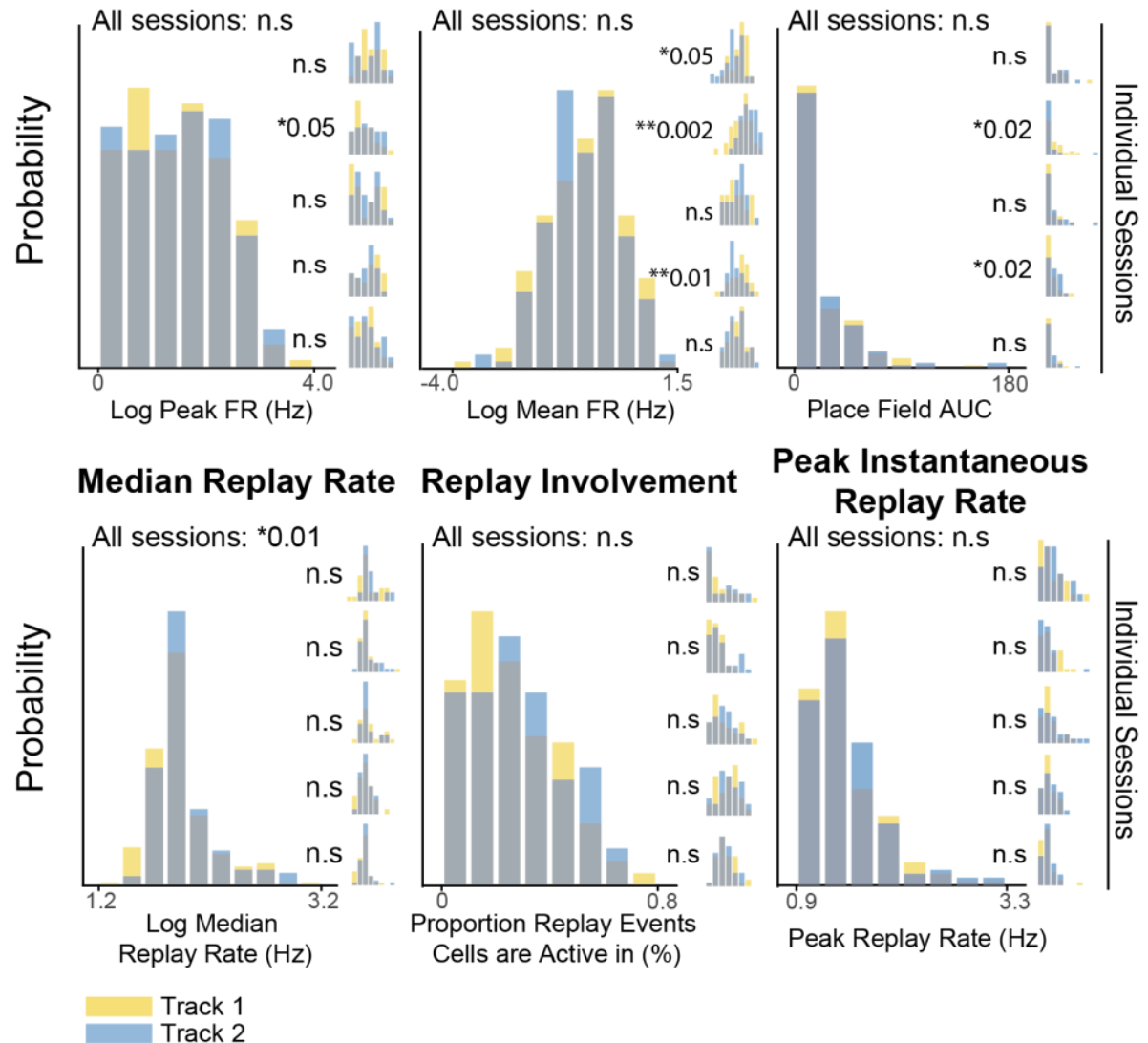

Peak Instantaneous

Replay Rate

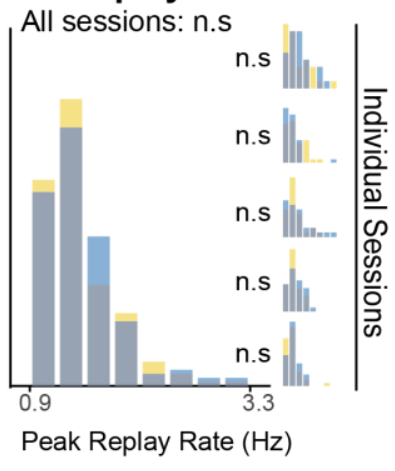

Fig. S5. Between track comparison of place field and replay properties. (A) Number of replay events (left) and replay event rate (right) for each track during PRE, RUN, and POST. The differences between tracks for any of these comparisons were not statistically significant (Wilcoxon sign rank, $P>0.05$ ). (B) Distribution of Peak firing rate on track (top left), mean firing rate on track (top center), place field AUC (top right), median replay rate (bottom left), replay involvement (bottom center) and peak instantaneous replay rate (bottom right) for tracks 1 and 2 , and for individual session distributions (inset) (K-S test, n.s. $P$ $>0.05)$. 

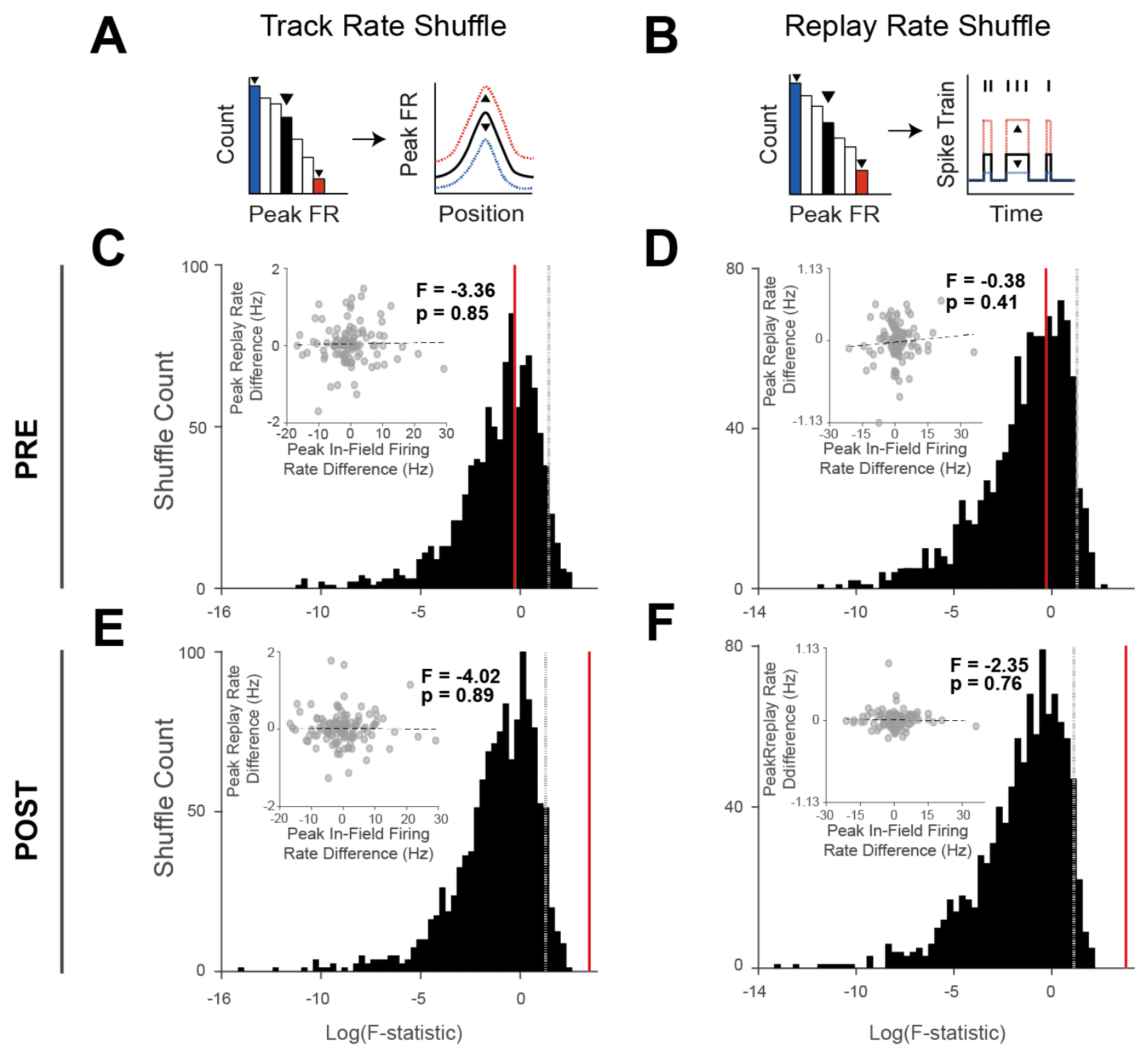

Fig. S6. Re-computing the statistical significance of regression analysis relative to a shuffled distribution. A Track rate shuffle (A) and Replay rate shuffle $(\mathbf{B})$ were used to randomize the overall firing rate of place fields within a track (x-axis) and the cell's firing rate during replay events (y-axis), respectively. An FStatistic distributions was then calculated to measure significance of the regression. (A and B) Example of the original rate (right plot, in black) randomly scaled up (right plot, in red) or down (right plot, in blue), after drawing a random value from the rate distribution (left plot). ( $\mathbf{C}$ to $\mathbf{F}$ ) F-statistic distributions obtained from shuffle (black), dashed gray line indicates $P<0.05$, and red line indicates F-Statistic of original data (main plot). One example of a regression plot within the shuffle distribution (inset plot). (C) PRE: Track rate shuffle. (D) PRE: Replay rate shuffle. (E) POST: Track rate shuffle. (F) POST: Replay rate shuffle. 


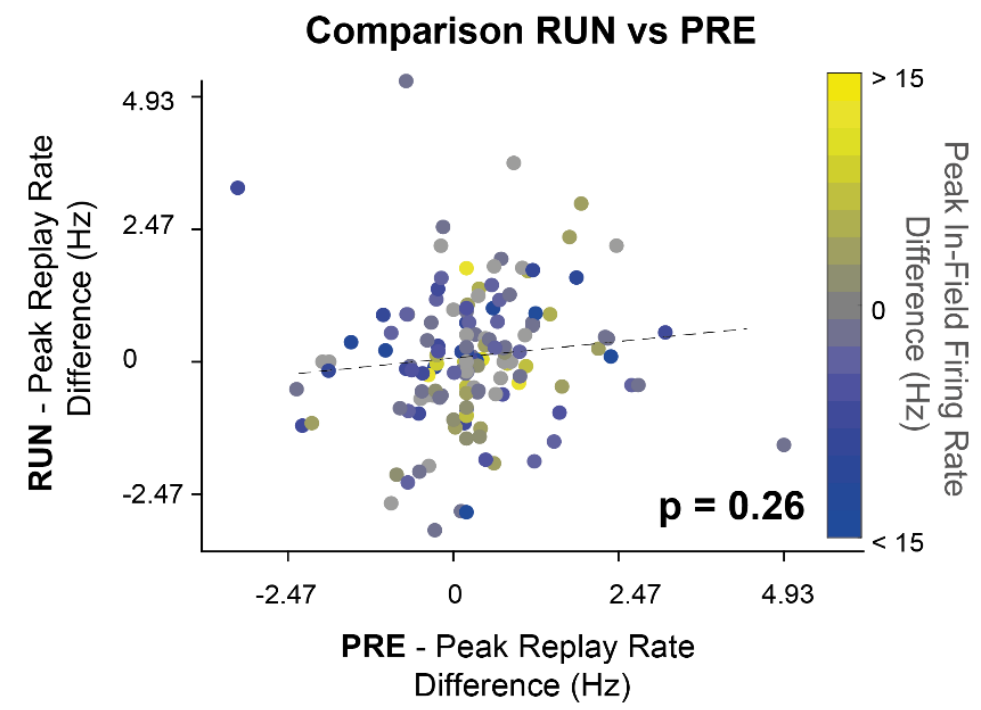

Fig. S7. Between track differences in replay rate during PRE is not predictive of replay rate differences observed during RUN local replay events. Peak replay rate difference for replay events (RUN vs. PRE), color coded by peak in-field rate differences. A linear regression was not statistically significant $(P>0.05)$. 


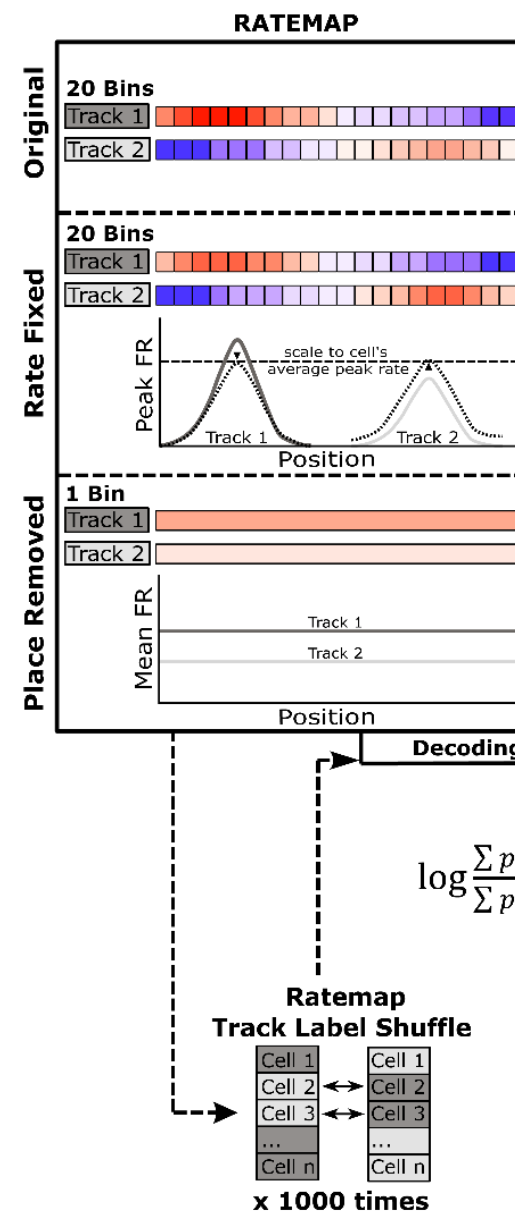

Fig. S8. Schematic of modified Bayesian decoding framework. The concatenated Track 1 and Track 2 ratemap (top first column) and spike counts during each replay event (top second column) are used for Bayesian decoding. This produces a posterior probabilistic distribution for each replay event, which is normalised across all position bins from both tracks to sum to 1 (Original, top row). Then, the relative probabilistic bias between Track 1 and Track 2 (Log odd) is computed. As control conditions, the original ratemap and replay spike counts are selectively modified such that only place information (Rate fixed manipulation, second row) or rate information (Place removed manipulation, third row) is available for Bayesian decoding. For Rate fixed manipulation, rate information is fixed by 1) rescaling each cell's peak in-field firing rate to the mean value across both tracks, and 2) rescaling its spike count during each replay event to the average firing rate across all replay events. For Place removed manipulation, the place and sequence information is removed by decoding using only a single position bin covering the entire track (when the animal's moving speed is above $5 \mathrm{~cm} / \mathrm{s}$ ) and a single time bin spanning the entire replay event. For both control conditions, the manipulated replay events are next decoded using the original decoding pipeline, including a Track 1 and Track 2 Ratemap Track label shuffle, obtained by randomly permuting each cell's Track 1 and Track 2 ratemaps 1000 times for each replay event (bottom left). The permutation is done by either keeping a cell's ratemap unaltered or performing a between track swap. The z-scored log odd for each replay event is obtained by comparing the raw log odd relative to the shuffled log odd distribution. For each behavioral epoch (i.e PRE, RUN and POST), Track 1 and Track 2 zscored log odd distributions are obtained by classifying each replay event according to the track identity determined by the sequenceness of the replay (bottom right). The track discrimination performance is quantified using two measurements. The first method quantifies binary discriminability using receiver operative characteristic curve (ROC curve). In brief, the ROC curve is constructed by plotting the true positive rate against false positive rates obtained by shifting discrimination threshold across the two z-scored log odd distributions. The area under the ROC curve (AUC) would thereby indicate the performance of binary discriminability where AUC $=1$ indicates perfect discrimination and $\mathrm{AUC}=0.5$ indicates chance level discrimination (right, top plot). The second method quantifies the mean 
difference between Track 1 and Track 2 z-scored log odd distributions. The mean z-scored log odd track difference for each behavioural epoch is obtained by taking the average of the z-scored log odd track difference across all replay events within each session and then across all five sessions. The statistical significance for this measurement is quantified using one-tailed p-value in which the z-scored log odd track difference is compared relative to a shuffled difference distribution where the track identity assigned to each replay event is randomized (right, bottom plot). 
Table S1. Overview of behavior, number of recorded cells and decoding accuracy for all sessions and rats.

\begin{tabular}{|c|c|c|c|c|c|c|c|c|c|}
\hline \multirow[b]{2}{*}{ SESSION } & \multirow[b]{2}{*}{ TRACK } & \multirow[b]{2}{*}{$\begin{array}{l}\text { NUMBER OF } \\
\text { LAPS }\end{array}$} & \multicolumn{2}{|c|}{$\begin{array}{l}\text { TIME RESTING } \\
\text { (min) }\end{array}$} & \multicolumn{3}{|c|}{ CELLS } & \multicolumn{2}{|c|}{ DECODING QUALITY (\%) } \\
\hline & & & PRE & POST & $\begin{array}{c}\text { Total } \\
\text { number of } \\
\text { cells on } \\
\text { track } \\
\end{array}$ & $\begin{array}{c}\text { Number of } \\
\text { cells } \\
\text { common to } \\
\text { T1 and T2 }\end{array}$ & $\begin{array}{l}\text { Included } \\
\text { cells in } \\
\text { replay } \\
\text { analysis } \\
\end{array}$ & $\begin{array}{c}\text { Classification } \\
\text { accuracy }\end{array}$ & $\begin{array}{c}\text { Local } \\
\text { decoding } \\
\text { accuracy }\end{array}$ \\
\hline Rat 1 & 1 & 30 & 72 & 76 & 42 & 27 & 27 & 91 & 81 \\
\hline Rat 1 & 2 & 20 & 72 & 76 & 44 & 27 & 27 & 97 & 82 \\
\hline $\begin{array}{c}\text { Rat 2, session } \\
1 \\
\end{array}$ & 1 & 15 & 59 & 125 & 70 & 40 & 40 & 87 & 86 \\
\hline $\begin{array}{c}\text { Rat 2, session } \\
1\end{array}$ & 2 & 59 & 59 & 125 & 63 & 40 & 40 & 80 & 89 \\
\hline $\begin{array}{c}\text { Rat 2, session } \\
2 \\
\end{array}$ & 1 & 22 & 54 & 104 & 49 & 28 & 28 & 92 & 82 \\
\hline $\begin{array}{c}\text { Rat 2, session } \\
2 \\
\end{array}$ & 2 & 33 & 54 & 104 & 43 & 28 & 28 & 90 & 79 \\
\hline $\begin{array}{c}\text { Rat 3, session } \\
1 \\
\end{array}$ & 1 & 34 & 49 & 123 & 59 & 33 & 33 & 94 & 85 \\
\hline $\begin{array}{c}\text { Rat 3, session } \\
1 \\
\end{array}$ & 2 & 27 & 49 & 123 & 51 & 33 & 33 & 94 & 80 \\
\hline $\begin{array}{c}\text { Rat 3, session } \\
2 \\
\end{array}$ & 1 & 36 & 69 & 137 & 72 & 46 & 46 & 94 & 85 \\
\hline $\begin{array}{c}\text { Rat 3, session } \\
2 \\
\end{array}$ & 2 & 38 & 69 & 137 & 57 & 46 & 46 & 97 & 86 \\
\hline
\end{tabular}




\begin{tabular}{|c|c|c|c|c|c|c|c|c|c|c|c|c|c|c|c|c|}
\hline & \multirow[b]{2}{*}{ Session } & \multicolumn{3}{|c|}{ Original } & \multicolumn{3}{|c|}{ Rate fixed } & \multicolumn{3}{|c|}{ Place removed } & \multicolumn{3}{|c|}{$\begin{array}{l}\text { Rate fixed, Place } \\
\text { randomized }\end{array}$} & \multicolumn{3}{|c|}{$\begin{array}{l}\text { Place removed, Rate } \\
\text { randomized }\end{array}$} \\
\hline & & Mean & $\mathbf{S E}$ & $P$ value & $\begin{array}{c}\text { Mea } \\
\text { n }\end{array}$ & $\mathbf{S E}$ & $P$ value & Mean & $\mathbf{S E}$ & $P$ value & Mean & $\mathbf{S E}$ & $P$ value & Mean & SE & $\begin{array}{c}\mathbf{P} \\
\text { value }\end{array}$ \\
\hline \multirow{6}{*}{$\frac{1}{2}$} & 1 & -0.11 & & 0.73 & -0.02 & & 0.56 & -0.02 & & 0.57 & 0.45 & & 0.05 & -0.30 & & 0.92 \\
\hline & 2 & 0.21 & & 0.15 & 0.32 & & 0.07 & 0.03 & & 0.39 & -0.21 & & 0.80 & -0.16 & & 0.77 \\
\hline & 3 & -0.33 & & 0.88 & -0.54 & & 0.96 & -0.35 & & 0.96 & 0.03 & & 0.46 & 0.02 & & 0.46 \\
\hline & 4 & 0.15 & & 0.32 & -0.21 & & 0.82 & 0.08 & & 0.24 & 0.03 & & 0.45 & -0.15 & & 0.73 \\
\hline & 5 & 0.50 & & 0.07 & 0.65 & & 0.02 & 0.01 & & 0.49 & 0.46 & & 0.12 & -0.25 & & 0.73 \\
\hline & Mean & 0.08 & 0.14 & 0.22 & 0.04 & 0.21 & 0.37 & -0.05 & 0.08 & 0.64 & 0.15 & 0.13 & 0.11 & -0.17 & 0.05 & 0.91 \\
\hline \multirow{6}{*}{ 孞 } & 1 & 2.51 & & $7.57 \times 10^{-27}$ & 2.13 & & $9.24 \times 10^{-26}$ & 1.15 & & $6.41 \times 10^{-19}$ & 0.01 & & 0.48 & -0.19 & & 0.87 \\
\hline & 2 & 3.22 & & $9.61 \times 10^{-36}$ & 2.37 & & $9.01 \times 10^{-28}$ & 1.39 & & $2.05 \times 10^{-28}$ & 0.48 & & 0.01 & 0.22 & & 0.10 \\
\hline & 3 & 2.55 & & $5.93 \times 10^{-23}$ & 2.37 & & $2.93 \times 10^{-22}$ & 1.27 & & $5.08 \times 10^{-18}$ & -0.19 & & 0.78 & 0.12 & & 0.22 \\
\hline & 4 & 2.50 & & $1.14 \times 10^{-22}$ & 2.44 & & $1.38 \times 10^{-22}$ & 0.37 & & $6.25 \times 10^{-06}$ & -0.11 & & 0.76 & -0.15 & & 0.79 \\
\hline & 5 & 3.09 & & $1.31 \times 10^{-29}$ & 2.26 & & $4.38 \times 10^{-25}$ & 1.52 & & $2.17 \times 10^{-20}$ & 0.20 & & 0.16 & 0.09 & & 0.29 \\
\hline & Mean & 2.78 & 0.16 & $1.08 \times 10^{-121}$ & 2.31 & 0.06 & $1.03 \times 10^{-101}$ & 1.14 & 0.20 & $9.23 \times 10^{-29}$ & 0.08 & 0.12 & 0.18 & 0.02 & 0.08 & 0.43 \\
\hline \multirow{6}{*}{ 봉 } & 1 & 0.84 & & $5.39 \times 10^{-11}$ & 0.43 & & $1.09 \times 10^{-05}$ & 0.32 & & $2.44 \times 10^{-05}$ & 0.17 & & 0.09 & 0.15 & & 0.18 \\
\hline & 2 & 1.53 & & $9.90 \times 10^{-35}$ & 1.03 & & $1.96 \times 10^{-24}$ & 0.45 & & $7.34 \times 10^{-19}$ & 0.33 & & 0.003 & 0.10 & & 0.15 \\
\hline & 3 & 1.63 & & $3.82 \times 10^{-19}$ & 1.44 & & $3.30 \times 10^{-16}$ & 0.84 & & $4.52 \times 10^{-18}$ & -0.14 & & 0.74 & -0.25 & & 0.98 \\
\hline & 4 & 0.70 & & $3.47 \times 10^{-08}$ & 0.78 & & $5.46 \times 10^{-11}$ & 0.20 & & $6.32 \times 10^{-06}$ & 0.20 & & 0.03 & 0.09 & & 0.20 \\
\hline & 5 & 1.79 & & $1.22 \times 10^{-34}$ & 1.59 & & $9.58 \times 10^{-30}$ & 0.89 & & $1.15 \times 10^{-21}$ & -0.12 & & 0.87 & 0.06 & & 0.30 \\
\hline & Mean & 1.30 & 0.22 & $1.03 \times 10^{-91}$ & 1.05 & 0.21 & $1.98 \times 10^{-74}$ & 0.54 & 0.14 & $8.12 \times 10^{-15}$ & 0.09 & 0.09 & 0.06 & 0.03 & 0.07 & 0.30 \\
\hline
\end{tabular}

Table S2. Summary statistics of the z-scored log odd difference per session and across sessions for PRE, RUN and POST. 


\begin{tabular}{|c|c|c|c|c|}
\cline { 3 - 4 } \multicolumn{2}{c|}{} & \multicolumn{3}{c|}{$\begin{array}{c}\text { VS Original 95\% confidence interval } \\
\text { BOLD values statistically significant from zero }\end{array}$} \\
\cline { 3 - 5 } & Mean AUC Diff & -0.04 & -0.02 & -0.05 \\
\hline \multirow{4}{*}{$\begin{array}{c}\text { Original VS Rate } \\
\text { fixed }\end{array}$} & SEM AUC Diff & 0.04 & 0.007 & 0.02 \\
\cline { 2 - 5 } & Lower CI & -0.12 & $\mathbf{- 0 . 0 3}$ & $\mathbf{- 0 . 0 8}$ \\
\cline { 2 - 5 } & Upper CI & 0.04 & $\mathbf{- 0 . 0 0 2}$ & $\mathbf{- 0 . 0 2}$ \\
\hline \multirow{4}{*}{$\begin{array}{c}\text { Original VS Place } \\
\text { removed }\end{array}$} & Mean AUC Diff & 0.06 & -0.19 & -0.10 \\
\cline { 2 - 5 } & SEM AUC Diff & 0.04 & 0.02 & 0.02 \\
\cline { 2 - 5 } & Lower CI & -0.02 & $\mathbf{- 0 . 2 2}$ & $\mathbf{- 0 . 1 3}$ \\
\cline { 2 - 5 } & Upper CI & 0.14 & $\mathbf{- 0 . 1 5}$ & $\mathbf{- 0 . 0 7}$ \\
\hline
\end{tabular}

Table S3. Summary statistics of the $95 \%$ confidence interval for the AUC difference between the original and the manipulated conditions. 


\begin{tabular}{|c|c|c|c|c|}
\cline { 3 - 4 } \multicolumn{2}{c|}{} & \multicolumn{3}{c|}{$\begin{array}{c}\text { VS Negative Control 95\% confidence interval } \\
\text { BOLD values statistically significant from zero }\end{array}$} \\
\cline { 3 - 5 } \multicolumn{2}{c|}{} & PRE & RUN & POST \\
\hline \multirow{2}{*}{$\begin{array}{c}\text { Rate fixed VS } \\
\text { Rate fixed } \\
\text { Place } \\
\text { randomized }\end{array}$} & Mean AUC Diff & -0.005 & 0.44 & 0.22 \\
\cline { 2 - 5 } & SEM AUC Diff & 0.04 & 0.02 & 0.02 \\
\cline { 2 - 5 } & Lower CI & -0.13 & $\mathbf{0 . 4 0}$ & $\mathbf{0 . 1 9}$ \\
\hline \multirow{2}{*}{$\begin{array}{c}\text { Place removed } \\
\text { VS Place } \\
\text { removed + Rate } \\
\text { randomized }\end{array}$} & Mean AUC Diff & 0.03 & $\mathbf{0 . 4 8}$ & $\mathbf{0 . 2 6}$ \\
\cline { 2 - 5 } & SEM AUC Diff & 0.07 & 0.26 & 0.14 \\
\cline { 2 - 5 } & Lower CI & -0.008 & 0.03 & 0.02 \\
\hline
\end{tabular}

Table S4. Summary statistics of the $95 \%$ confidence interval for the AUC difference between the manipulated conditions and their respective negative controls. 
bioRxiv preprint doi: https://doi.org/10.1101/2021.07.15.452506; this version posted September 25, 2021. The copyright holder for this preprint (which was not certified by peer review) is the author/funder, who has granted bioRxiv a license to display the preprint in perpetuity. It is made available under aCC-BY-NC-ND 4.0 International license.

\begin{tabular}{|c|c|c|}
\cline { 2 - 3 } \multicolumn{1}{c|}{} & \multicolumn{2}{c|}{$\begin{array}{c}\text { DeLong Test VS Original (Significance } \mathbf{0 . 0 5} \text { ) } \\
\text { BOLD values statistically significant }\end{array}$} \\
\hline & Original VS Rate fixed & Original VS Place removed \\
\hline PRE & 0.15 & 0.15 \\
\hline RUN & $\mathbf{0 . 0 0 7}$ & $\mathbf{5 . 5 6} \times \mathbf{1 0}^{-34}$ \\
\hline POST & $\mathbf{8 . 1 5 \times 1 0 ^ { - 0 8 }}$ & $\mathbf{6 . 3 3} \times \mathbf{1 0}^{-12}$ \\
\hline
\end{tabular}

Table S5. Summary statistics of the DeLong Test comparing the ROC curves between the original and the manipulated condition. 


\begin{tabular}{|c|c|c|}
\cline { 2 - 3 } \multicolumn{1}{c|}{} & \multicolumn{2}{c|}{$\begin{array}{c}\text { DeLong Test VS Negative control (Significance = 0.05) } \\
\text { BOLD values statistically significant }\end{array}$} \\
\hline PRE & $\begin{array}{c}\text { Rate fixed VS Rate fixed + } \\
\text { Place randomized }\end{array}$ & $\begin{array}{c}\text { Place removed VS Place } \\
\text { removed + Rate randomized }\end{array}$ \\
\hline RUN & 0.91 & 0.23 \\
\hline POST & $\mathbf{2 . 2 8 \times 1 0 ^ { - 9 8 }}$ & $\mathbf{7 . 1 4 \times 1 0 ^ { - 8 8 }}$ \\
\hline
\end{tabular}

Table S6. Summary statistics of the DeLong Test comparing the ROC curves between the manipulated conditions and their respective negative controls. 